\title{
Magnetic reconnection during steady magnetospheric convection and other magnetospheric modes
}

\author{
Benoit Hubert $^{1}$, Jean-Claude Gérard ${ }^{1}$, Steve E. Milan ${ }^{2}$, and Stanley W. H. Cowley ${ }^{2}$ \\ ${ }^{1}$ Laboratory for Planetary and Atmospheric Physics, University of Liège, Liège, Belgium \\ ${ }^{2}$ Department of Physics and Astronomy, University of Leicester, Leicester, UK \\ Correspondence to: Benoit Hubert (b.hubert@ulg.ac.be)
}

Received: 14 December 2016 - Revised: 21 February 2017 - Accepted: 23 February 2017 - Published: 28 March 2017

\begin{abstract}
We use remote sensing of the proton aurora with the IMAGE-FUV SI12 (Imager for Magnetopause to Aurora Global Exploration-Far Ultraviolet-Spectrographic Imaging at $121.8 \mathrm{~nm}$ ) instrument and radar measurements of the ionospheric convection from the SuperDARN (Super Dual Aurora Radar Network) facility to estimate the open magnetic flux in the Earth's magnetosphere and the reconnection rates at the dayside magnetopause and in the magnetotail during intervals of steady magnetospheric convection (SMC). We find that SMC intervals occur with relatively high open magnetic flux (average $\sim 0.745 \mathrm{GWb}$, standard deviation $\sim 0.16 \mathrm{GWb}$ ), which is often found to be nearly steady, when the magnetic flux opening and closure rates approximately balance around $55 \mathrm{kV}$ on average, with a standard deviation of $21 \mathrm{kV}$. We find that the residence timescale of open magnetic flux, defined as the ratio between the open magnetospheric flux and the flux closure rate, is roughly $4 \mathrm{~h}$ during SMCs. Interestingly, this number is approximately what can be deduced from the discussion of the length of the tail published by Dungey (1965), assuming a solar wind speed of $\sim 450 \mathrm{~km} \mathrm{~s}^{-1}$. We also infer an enhanced convection velocity in the tail, driving open magnetic flux to the nightside reconnection site. We compare our results with previously published studies in order to identify different magnetospheric modes. These are ordered by increasing open magnetic flux and reconnection rate as quiet conditions, SMCs, substorms (with an important overlap between these last two) and sawtooth intervals.
\end{abstract}

Keywords. Magnetospheric physics (auroral phenomena; magnetospheric configuration and dynamics; solar windmagnetosphere interactions)

\section{Introduction}

The solar wind plasma outflow from the Sun carries the interplanetary magnetic field (IMF) outward into the solar system, where it interacts with the Earth's magnetic field. When the IMF is oriented southward, reconnection of the geomagnetic field with the IMF at the outer magnetopause boundary of the planetary field efficiently produces open magnetic field lines which map from the polar regions of the planet into the solar wind. These open-field lines are dragged anti-sunward by the solar wind flow and are stretched into a long magnetic tail, in which the field lines eventually reconnect and return toward the Earth, back to the dayside (Dungey, 1961). This sequence of processes broadly governs the interaction between the solar wind and the Earth's magnetosphere-ionospherecoupled system, including the overall convection (Cowley and Lockwood, 1992, and references therein). This interaction constrains the evolution of the magnetospheric system that is known to be able to produce several possible responses, the most important of which being the auroral substorm cycle, which classically consists of a growth phase, a substorm onset, an expansion phase and finally a recovery phase (Akasofu, 1964; McPherron, 1970). During the growth phase, the interplanetary magnetic field (IMF) carried by the solar wind is usually oriented southward so that it efficiently reconnects with the geomagnetic field, producing new open flux. This phase ends in a substorm onset characterized by a sudden localized brightening of the polar aurora near midnight, which announces the expansion phase during which accumulated open flux is closed by intense magnetic reconnection in the magnetotail (Milan et al., 2004, 2007, and references therein). The magnetosphere then returns to a less active state during the recovery phase, and the flux closure rate 
decreases. During the substorm cycle, the open magnetic flux in the magnetosphere, i.e. the flux of field lines that close in the interplanetary medium (the magnetic field remains divergenceless), is often found to lie between $\sim 0.6$ and $0.7 \mathrm{GWb}$. It can also reach $\sim 0.8 \mathrm{GWb}$ and sometimes more, while the reconnection rate can become as large as $\sim 120 \mathrm{kV}$ (Hubert et al., 2006a; Milan et al., 2009; Boakes et al., 2009).

However, when the merging rate of the IMF and the geomagnetic field becomes very large, the evolution of the magnetosphere can depart from the pattern of the substorm cycle. The magnetosphere then exhibits another response and enters an interval of "sawtooth" events. During a sawtooth interval, vigorous magnetic reconnection on the dayside causes the magnetosphere to accumulate up to $\sim 1.2 \mathrm{GWb}$ of open magnetic flux and sometimes more (De Jong et al., 2007; Hubert et al., 2008), while the reconnection rate can easily exceed 130-150 kV (Hubert et al., 2008), whereas during the substorm cycle, the open magnetic flux rarely exceeds $1 \mathrm{GWb}$ with generally lower reconnection rates. The other extreme situation happens at quiet times, generally when the IMF is oriented northward so that dayside magnetic reconnection relies on the By component of the IMF (instead of $B_{z}$ when the IMF is southward), producing a much lower merging rate. During such quiet times, the open magnetic flux becomes smaller but nevertheless remains non-zero $(\sim 0.4-0.5 \mathrm{GWb}$ and less) and still depends on the competing opening and closure rates of magnetic flux by dayside and nightside reconnection (respectively) that typically amount to less than $\sim 40 \mathrm{kV}$ (Hubert et al., 2006a).

Steady magnetospheric convection (SMC) intervals represent a fourth distinct situation in which the solar wind interaction produces near-steady conditions. They are considered representative of the steady state of the magnetosphere interacting with the solar wind. SMCs have moderate reconnection rates; they differ from the classical substorm cycle and from the quiet-time situation during which the IMF-magnetosphere interaction is reduced. The properties of SMC intervals have been thoroughly reviewed by Sergeev et al. (1996), and we will only give a short summary in this paragraph. During SMC intervals, the magnetospheric convection is stable, governed by magnetic reconnection that produces open magnetic flux along the dayside magnetopause and closes previously opened magnetic flux in the magnetotail. Anti-sunward transport carries open-field lines to a reconnection site located as far as, probably, $\sim 50$ 100 Earth radii downtail (Sergeev et al., 1996, and references therein). The tail configuration is then intermediate between a growth phase configuration near the Earth within $\sim 12 R_{\mathrm{E}}$ downtail and a recovery phase configuration farther downtail. Open magnetic flux is introduced in the system on the dayside and transported downtail like during a growth phase. However, sustained flux closure takes place in the more distant tail that prevents a large accumulation of open magnetic flux, unlike during a growth phase. This can give the tail a configuration similar to what can be found at the end of a sub- storm expansion and during the recovery phase, when flux closure still takes place while the amount of open magnetic flux is not as large as prior to the substorm onset. Indeed, detailed analysis of in situ tail data and modelling indicate a very tail-like magnetic field configuration and a very intense and thin current sheet in the near-Earth tail reminiscent of substorm growth phase, whereas large magnetic field values are found in the mid-tail along with an expanded plasma sheet, which resembles recovery phase conditions (Sergeev et al., 1996). In addition, the convection has a component towards the tail flanks in the plasma sheet and transient activations, possibly reminiscent of "bursty bulk flows" (BBF), can also take place (Sergeev et al., 1996, and references therein). It is generally expected that the open magnetic flux content of the magnetosphere should not vary by more than 10 $20 \%$ during SMCs and that this state should last for more than $\sim 4-6 \mathrm{~h}$, i.e. significantly longer than the characteristic timescale of the substorm cycle. During such an interval, the energy input from the solar wind into the magnetosphere is rather large and fairly constant. In principle, there is no obvious physical reason to assume that there would be a lower limit to the cross-polar cap potential for SMCs to occur; i.e. in principle, a steady state could be achieved by the magnetosphere whatever the value of the IMF $B_{z}$. However, Sergeev et al. (1996, and references therein) reported that SMCs are sometimes recorded during intervals when the cross-polar cap potential drop, which can differ from the reconnection rate, ranges between 60 and $90 \mathrm{kV}$, i.e. values compatible with the development of substorm growth and expansion phases that mostly take place when the IMF is oriented southward with a large $B_{z}$ component (although magnetic merging can also take place due to the presence of an IMF $B_{y}$ component, as already mentioned above). The reason why the steady state is mostly achieved during active intervals (except for northward IMF quiet-time conditions) still needs to be explained. Recently, Walach et and Milan (2015) showed that a large fraction of SMCs can be viewed as "driven expansion phases", i.e. where the IMF remains southwards for a prolonged time interval after substorm onset.

Tanskanen et al. (2005) also studied SMC intervals (which they called continuous magnetospheric dissipation events) discriminating them from other magnetospheric modes by the steadiness of the total pressure in the tail. In that study, intervals were considered to be SMC events when the total pressure did not increase by more than a factor of 2 nor decrease by more than a factor of 2 , distinguishing them from loading intervals, during which the pressure could increase by more than a factor of 2 , and unloading intervals, during which the total pressure could decrease by more than a factor of 2. This approach thus relates to energy loading and unloading of the magnetosphere if we consider the total pressure as an energy density. These authors also pointed out that BBFs occur more often and at a lower velocity during SMC intervals than during unloading intervals. 
Juusola et al. (2013) studied the relation between SMCs and the ring current. They found that the ring current is generally enhanced during SMC intervals and has a stabilizing effect that prevents near-Earth reconnection to occur. They also found that the solar wind driving before and during the SMC interval can be similar, the SMC mode appearing when the ring current reaches a sufficiently large value.

Kissinger et al. (2012a, b) showed that SMC intervals are nearly always preceded by a substorm expansion, which sets up SMC-specific conditions in the magnetotail: the total pressure is enhanced and the earthward return flow is then diverted along the tail flanks, transporting towards the dayside newly closed magnetic flux produced along a neutral line located downtail at a distance of between 35 and 45 Earth radii. This suggests a possible preconditioning of the magnetosphere by preceding geomagnetic conditions before an SMC can occur.

Since diagnosing stable conditions in magnetospheric convection from ionospheric data alone has long been challenging, SMC intervals have often been identified based on a set of criteria. First, stable, continuously southward IMF should be present for more than 4-6h. Second, enhanced convection must occur during the interval,producing increased levels of the auroral upper (AU) and auroral lower (AL) indices (Davis and Sugiura, 1966), with the auroral electrojet (AE) index $>200 \mathrm{nT}$. Third, no substorm signatures should be observed on the ground during the SMC interval. Fourth, no current sheet disruption or plasmoid ejection in the nearEarth magnetotail should be recorded, i.e. no substorm expansion signatures in the tail (Sergeev and Lennartson, 1988; Sergeev et al., 1996).

The relation between $\mathrm{AE}$ and the steady convection state has been used by De Jong and Clauer (2005) to identify SMCs. These authors proposed that any interval with $\mathrm{AE}>200 \mathrm{nT}$, no substorm signatures and a stable polar cap area (so that the dayside and nightside magnetic reconnection rates were roughly in balance) can be considered an SMC. De Jong et al. (2007) analysed a set of SMC intervals whose selection was based on their stable polar cap area, high activity $(\mathrm{AE}>200 \mathrm{nT})$ and the absence of substorm signatures. They used remote sensing of the global aurora to compare the magnetospheric open flux during SMCs, sawtooth events and substorms. They found that the polar cap is fairly stable during SMCs, in contrast with substorms and sawtooth intervals, during which it varies significantly. McWilliams et al. (2006) studied SMC intervals selected using the criterion of O'Brien et al. (2002) and analysed ionospheric convection data from the Super Dual Aurora Radar Network (SuperDARN). They suggested that the convection pattern was consistent with the production of open flux in the tail lobes resulting from dayside magnetic reconnection occurring at a prenoon, high-latitude Southern Hemisphere merging site. They also showed the formation of a double auroral oval during an SMC case study (another feature common to SMCs), which points to two regions of upward field-aligned current poleward and equatorward of a downward field-aligned current, consistent with the vorticity of the ionospheric convection recorded using SuperDARN. McWilliams et al. (2006) also analysed in situ particle data from the Defense Meteorological Satellite Program (DMSP) showing signatures of magnetic reconnection on the dayside. They suggested, based on the large extent of the dayside merging site inferred from the DMSP data, that intense reconnection must take place on the dayside magnetopause, compensated for by flux closure in the tail.

We have developed a method that combines SuperDARN observations of ionospheric convection with global images of the proton aurora recorded with the Spectrographic Imaging at $121.8 \mathrm{~nm}$ (SI12) instrument (Mende et al., 2000a, b) onboard the Imager for Magnetopause to Aurora Global Exploration (IMAGE) satellite, to estimate the open magnetic flux threading the polar cap and the reconnection rates at the dayside magnetopause and in the magnetotail (Hubert et al., 2006a). These rates are expressed as electric voltages from the application of Faraday's law $\left(1 \mathrm{~Wb} \mathrm{~s}^{-1}=1 \mathrm{~V}\right)$. Since the SI12 images are almost uncontaminated by dayglow, the method has the advantage of working during any season. The method has been validated against DMSP observations by Hubert et al. (2006a), who also applied the method to several substorm intervals, showing the flux opening and closure associated with the growth and expansion phases of the substorm cycle. They also showed that magnetic flux is closed by pseudo-breakups and that poleward boundary intensifications (PBIs) observed during the recovery phase result from minor reactivations of flux closure in the tail. In addition to the DMSP validation done by Hubert et al. (2006a), Hubert et al. (2010) compared the global-scale boundary determination from the IMAGE-FUV (Far Ultraviolet)-SI12 observations with the polar cap boundary location determined locally at a higher space and time resolution using the European Incoherent Scatter Scientific Association (EISCAT) facility. Both methods showed an acceptable agreement: for most of the analysed intervals, the polar cap boundary determined by both methods is collocated within the space resolution of both methods, i.e. $\sim 0.5^{\circ}$ MLAT on average (this number was found to depend on substorm phase; we refer the interested reader to the original study). Hubert et al. (2010) also show an interval for which the discrepancy can reach $\sim 1.9^{\circ}$ MLAT on average, which remains relatively good considering that the space resolution of the SI12 instrument is on the order of $1^{\circ}$ MLAT. These authors also undertook a comparison with the location of the magnetic convection reversal boundary (MCRB) and with the boundary location found from observation of the $\mathrm{OI}\left({ }^{1} \mathrm{D}\right)$ emission at $630 \mathrm{~nm}$. Here again a satisfactory agreement was found: the SI12 and MCRB boundary were generally, but not always, collocated considering the resolution of both methods except for notable intervals showing large discrepancies (not larger than $4^{\circ}$ MLAT), especially close to the Harang discontinuity where the MCRB does not necessarily follow the vicinity of the polar cap boundary. 
The method has also been applied to the study of flux closure induced by solar wind dynamic pressure pulses (Hubert et al., 2006b, 2009), showing that a strong compression of the tail by a solar wind pressure discontinuity extends to the plasma sheet and stimulates the process of flux closure. Hubert et al. (2007) analysed the magnetic reconnection associated with auroral streamers, i.e. the ionospheric counterpart of BBFs, and proposed that these events are initially produced by flux closure, possibly followed by subsequent acceleration by other processes. Using the same method, $\mathrm{Hu}-$ bert et al. (2008) showed, as already outlined above, that sawtooth events take place when the magnetosphere is overloaded by open magnetic flux produced by magnetic reconnection with the IMF at very high rates on the dayside, faster than it can be closed by the usual substorm cycle, such that the magnetosphere accumulates a large amount of open magnetic flux, which is eventually closed at a high rate in the tail.

The FUV experiment onboard the IMAGE satellite not only provides images of the proton aurora. The Wideband Imaging Camera (WIC) observes the aurora between 120 and $200 \mathrm{~nm}$, while the Spectrographic Imaging at $135.6 \mathrm{~nm}$ instrument (SI13) is sensitive between $\sim 130$ and $\sim 140 \mathrm{~nm}$ (Mende et al., 2000a, b). Both instruments provide images of auroral emissions mainly excited by secondary electrons produced by both the electron and proton precipitation, the most important of which are the OI 130.4 and $135.6 \mathrm{~nm}$, NI $149.2 \mathrm{~nm}$, and $\mathrm{N}_{2}$ LBHLyman-Birge-Hopfield (LBH) emissions. The three FUV imagers, namely WIC, SI13 and SI12, can be used simultaneously to monitor the evolution of the aurora and to identify the commencement of an expansion phase, for example. It must be noted that dayglow luminescence is excited by photoelectrons, which are produced in the same kinetic energy range as the auroral secondary electrons, so that both the WIC and SI13 instruments are sensitive to dayglow. By contrast, the SI12 imager detects Doppler-shifted Lyman- $\alpha$ photons emitted by precipitating protons that have captured an electron, so that it is nearly insensitive to dayglow emissions, the instrument being designed to efficiently reject the nearby geocoronal HI-Lyman$\alpha 121.6 \mathrm{~nm}$ and NI $-120 \mathrm{~nm}$ emissions. The choice by $\mathrm{Hu}-$ bert et al. (2006a) to avoid using the data from the WIC and SI13 instruments in the estimation of the polar cap boundary location was not justified by the sensitivities of the WIC and SI13 instruments, which are very good too. It rather relied on the need to use dayside auroral images to estimate the open magnetic flux and reconnection voltages in all seasons, including when the dayside aurora is largely immersed in the dayglow, where the Poisson noise of the images can easily dominate the auroral signal. In principle, the WIC and SI13 images could thus also be used to study the polar cap boundary on the nightside. However, we focus on the SI12 observation of the proton aurora because we are interested in both the dayside and nightside parts of the oval.

In this study, we analyse several SMC intervals previously identified by McWilliams et al. (2006) and De Jong et al. (2007), for which good quality (i.e. with convenient viewing geometry and auroral intensity) SI12 images of the proton aurora are available simultaneously with SuperDARN measurement of the ionospheric convection. We combine the SI12 images and the SuperDARN data to estimate the dayside and nightside magnetic reconnection rates and the open magnetic flux using the method described and validated by Hubert et al. (2006a). Our scientific objective is not only to characterize the magnetic reconnection process during SMC intervals but also to compare our results with other magnetospheric situations, namely quiet time, substorm cycle and sawtooth events, and to determine the ranges of open magnetic flux and reconnection rates relevant to those wellidentified magnetospheric modes. We thus finally discuss our results in the context of our previous studies, presenting quiet times, the auroral substorm cycle, SMC intervals and sawtooth events as four magnetospheric modes which occur depending on the input provided by the solar wind and the accumulated open magnetic flux.

\section{Case studies}

We have analysed a set of 11 intervals during which SMC conditions occur. These intervals encompass a total of $\sim 1700$ IMAGE-FUV images representative of SMC conditions. In Sect., 2.1, 2.2 and 2.3, we will first present three SMC cases in detail. Eight other SMC intervals will be briefly described in Sect. 2.4, concentrating on the modest differences existing in these eight cases compared with those of Sect. 2.1, 2.2 and 2.3.

\subsection{SMC interval on 3 January 2001}

An SMC interval was identified by McWilliams et al. (2006) on the 3 January 2001 between 02:57 and 06:46 UT, at the end of a substorm expansion phase with onset at 02:35 UT. The interval was identified based on the AE $>200 \mathrm{nT}$ criterion and imposing that the decrease rate of $\mathrm{AL}$ was less than $25 \mathrm{nT} \mathrm{min}{ }^{-1}$ to ensure no substorm onset. The magnitude of both the AL and AU indices increases after 02:30 UT indicating a progressive increase in the convection, together producing an AE index larger than $400 \mathrm{nT}$ (Fig. 1). The three indices indicate significant activity, but this differs from that of the substorm cycle. We also verified from the detailed magnetograms from the Fennoscandian International Monitor of Auroral Geomagnetic Effecs (IMAGE, not to be confused with the IMAGE satellite) magnetometer chain and from the Canadian Array for Realtime Investigations of Magnetic Activity (CARISMA) network (Mann et al., 2008) that no clear substorm signatures are present (not shown). Indeed, the AE, $\mathrm{AU}$ and $\mathrm{AL}$ indices are composite quantities that summarize the data from a large number of magnetometers distributed in longitude, and one should not expect to find a signature from a sharp substorm expansion in magnetograms when these 

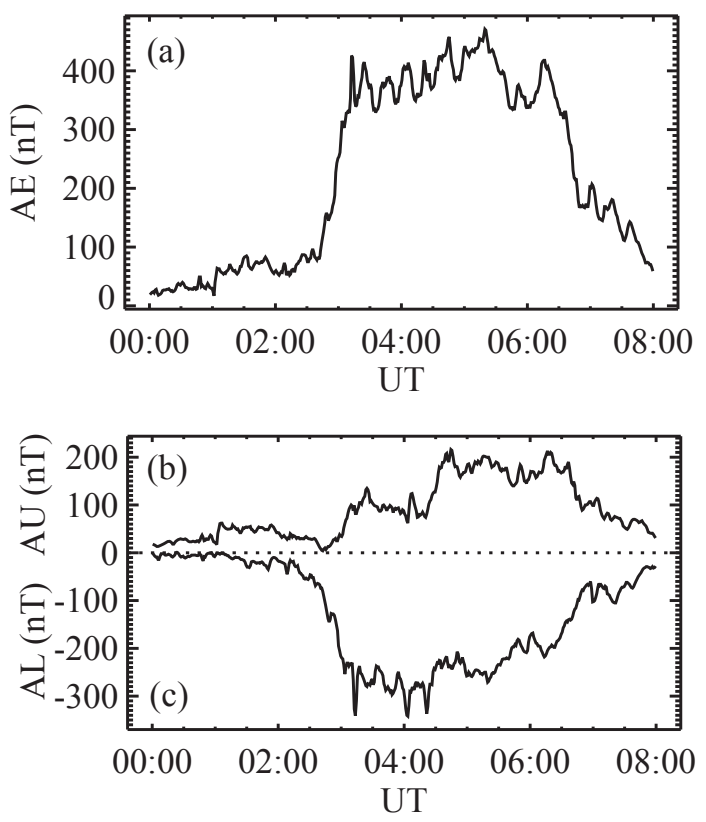

Figure 1. (a) AE, (b) AL and(c) AU indices recorded on 3 January 2001 .

indices reveal none. The IMAGE-FUV instruments provide auroral images between 00:00 and 05:41 UT, showing that the auroral activity was high after the onset at 02:35 UT and during the following SMC interval. The solar wind properties remained fairly stable during a long interval that day, as shown in Fig. 2. The solar wind velocity varied very slowly from $\sim 330$ to $\sim 350 \mathrm{~km} \mathrm{~s}^{-1}$ over $\sim 5.5 \mathrm{~h}$, i.e. an average rate of variation of only $\sim 0.001 \mathrm{~km} \mathrm{~s}^{-2}$. The solar wind dynamic pressure $P_{\text {dyn }}$ was more variable, due to the variations in the solar wind density. The IMF remained southward for $\sim 5 \mathrm{~h}$, allowing the magnetospheric and interplanetary magnetic fields to merge and produce open magnetic flux at the dayside magnetopause. It is thus not surprising that the observed aurora remained active for a long interval.

SuperDARN observations of the ionospheric convection were also available simultaneously with the IMAGE-FUV observations and were used to reconstruct the ionospheric electric field in the polar cap, which is needed to allow us to estimate the opening $\left(V_{\mathrm{op}}\right)$ and closure $\left(V_{\mathrm{cl}}\right)$ electric potentials that represent the rates of magnetic reconnection (Hubert et al., 2006a). The open magnetic flux $(\Phi)$ threading the polar cap, delineated using the SI12 observations of the proton aurora, was also estimated (Fig. 3a). The open magnetic flux increases after 01:00 UT due to reconnection with the IMF. The reconnection at the magnetopause is so efficient that the flux closure associated with the expansion phase observed after 02:35 UT does not prevent the open magnetic flux from increasing. Figure $3 b$ shows several SI1 2 images in polar view, obtained during that interval, with the estimated location of the polar cap boundary overlaid. This boundary

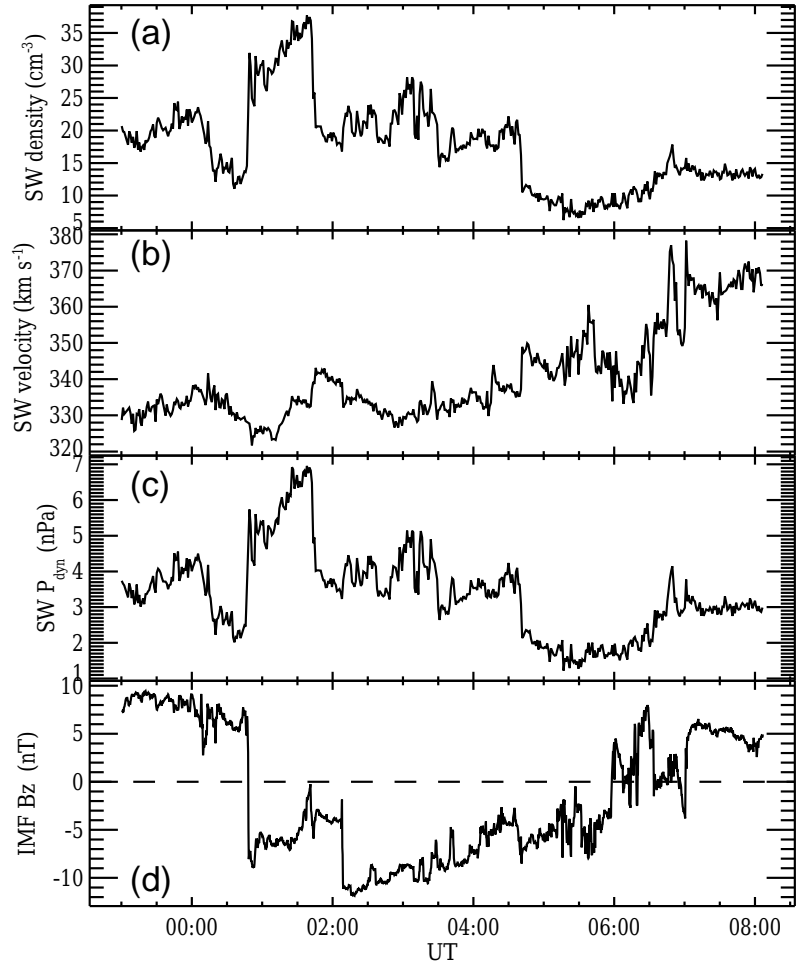

Figure 2. Solar wind properties recorded by the ACE (Advanced Composition Explorer) satellite on 2 and 3 January 2001. The timescale has been shifted by $79 \mathrm{~min}$ to approximately account for the propagation time from the spacecraft to the Earth. The solar wind dynamic pressure in (c) is computed from the number density and the velocity shown in (a) and (b). The IMF remained southward for $\sim 5 \mathrm{~h}$, as shown in (d) (GSM coordinates).

is represented using a Fourier series, allowing interpolation in unconstrained MLT sectors (i.e. MLT sectors where the aurora is too dim to be detected with the SI12 instrument so that the location of the boundary cannot be determined from observation). This choice also has another advantage: when the magnetic field can be assumed to be dipolar (which is certainly the case at ionospheric altitudes for the purpose of computing the magnetic flux), the magnetic flux threading the polar cap (i.e. the open magnetic flux) delineated by such a Fourier series can be easily computed analytically (Appendix A). The auroral brightness obviously increases as the activity rises, first slowly between 00:01 and $\sim 02: 30$ and more sharply thereafter to become very bright after 03:00. The polar cap area nevertheless remains roughly constant between 03:00 and 04:00 UT. The auroral brightness is even larger after 04:00, when flux closure reduces the magnetospheric open flux. Apparent fluctuations of the boundary appear versus UT and are also present versus MLT (although it does not appear in the figure). These fluctuations are efficiently filtered out using an appropriate time smoothing over a timescale of $\sim 6$ min full width at half maximum (FWHM). The smoothing is realized by applying successive boxcar av- 
eraging with decreasing width. This procedure fairly mimics convolution by a Gaussian function but with a smaller computational cost. In this study, we use widths varying from 3 to 6 (with steps of 1), and when the width is an even number, the next larger odd number is used instead. Tests already conducted by Hubert et al. (2006a) showed that this procedure efficiently smooths time variations of the estimated open magnetic flux occurring at a too large rate to be physically meaningful. On the other hand, the time-smoothed Fourier series representing the polar cap boundary is less able to correctly account for short-lived real variations of the polar cap boundary taking place on a small MLT scale. The increase (decrease) of the polar cap area associated with net flux opening (flux closure) is conspicuous over the interval, comparing the polar cap boundary prior to and after 03:00 UT (04:00 UT, respectively).

The ionospheric convection velocity vectors deduced from the SuperDARN data are also overlaid in each image, after averaging on a $5^{\circ}$ MLAT $\times 2 \mathrm{~h}$ MLT grid for the sake of readability of the figure. Despite local variations of the velocity field, the broad brush properties of the convection pattern remain rather stable during the SMC interval, especially between 03:00 and 04:00 UT, with an important westeast component in the midnight sector and a fairly stable return flow observed in the dawn sector. It must, however, be stressed that it is not easy to follow the convection pattern using the SuperDARN radar network during an extended interval because the MLAT-MLT region covered by the observations changes as the planet rotates, no radar being available across the Siberian region. Figure 4a shows our estimated net reconnection voltage, which gives the time derivative of the open magnetic flux. As expected, the net voltage is significantly positive when the open magnetic flux grows at a sustained rate between 01:00 and 03:00 UT. After 03:00 UT, the net voltage is close to zero, and the open magnetic flux remains fairly constant for $\sim 1 \mathrm{~h}$. This stability of the open magnetic flux can be considered a symptomatic feature of SMCs, as it was proposed earlier by Yahnin et al. (1994), Sergeev et al. (1996), De Jong and Clauer (2005), and De Jong et al. (2007). Sergeev et al. (1996) also pointed out that a considerable amount of open magnetic flux had to be closed in the magnetotail during SMCs. Figure $4 \mathrm{~b}$ and $\mathrm{c}$ show that the reconnection rate between the IMF and the geomagnetic field is large at the time the open flux grows, peaking above $120 \mathrm{kV}$, whereas it returns to smaller though still large values around $\sim 50 \mathrm{kV}$ between 03:00 and 04:00 UT, when the open flux is steady. The flux closure voltage reaches $\sim 80 \mathrm{kV}$ during the expansion phase starting at 02:35 UT, which is lower than that often found during a substorm expansion, when a flux closure rate peaking above $100 \mathrm{kV}$ is not uncommon. The relatively low value of the closure voltage during this expansion phase is consistent with the open flux still increasing at that time, the net reconnection rate remaining positive until 03:05 UT. Between 03:00 and 04:00 UT, when the open magnetic flux remains fairly steady, the flux closure
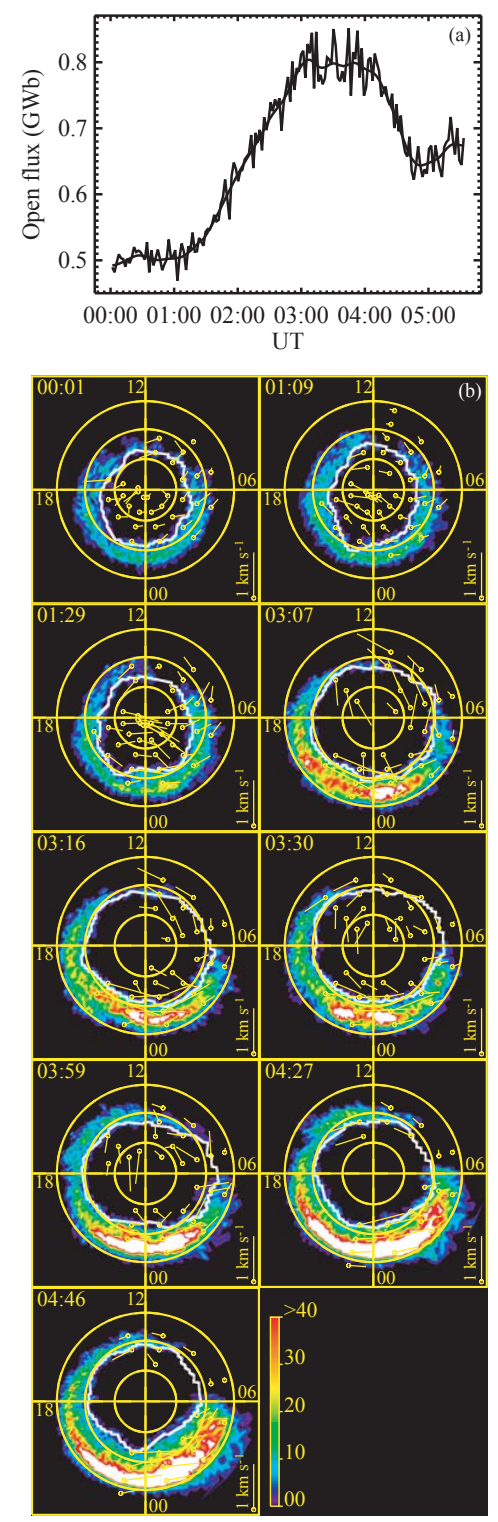

Figure 3. (a) Open magnetic flux estimated using IMAGE SI12 observations of the proton aurora on 3 January 2001. (b) Polar view of the proton aurora observed with the SI12 instrument, shown in geomagnetic coordinates. The colour scale is expressed in counts. Concentric circles are $10^{\circ}$ MLAT apart and noon is at the top of each snapshot (12 h MLT). The ionospheric convection velocity vector field deduced from SuperDARN measurements is also plotted, averaged on a $5^{\circ}$ MLAT $\times 2 \mathrm{~h}$ MLT grid. A $1 \mathrm{~km} \mathrm{~s}^{-1}$ reference arrow is shown in the lower right corner of each picture. The average of the SuperDARN measurements of the ionospheric velocity over the polar caps shown is, in chronological order, 248, 247, 442, 594, 536, $502,467,496$ and $462 \mathrm{~m} \mathrm{~s}^{-1}$. The location of the polar cap boundary deduced from the SI12 images and represented with a Fourier series is overlaid. Uncertainties in the polar cap boundary location produce artificial noisy variations of the estimated open magnetic flux (a), which can be eliminated by applying appropriate smoothing as shown. 

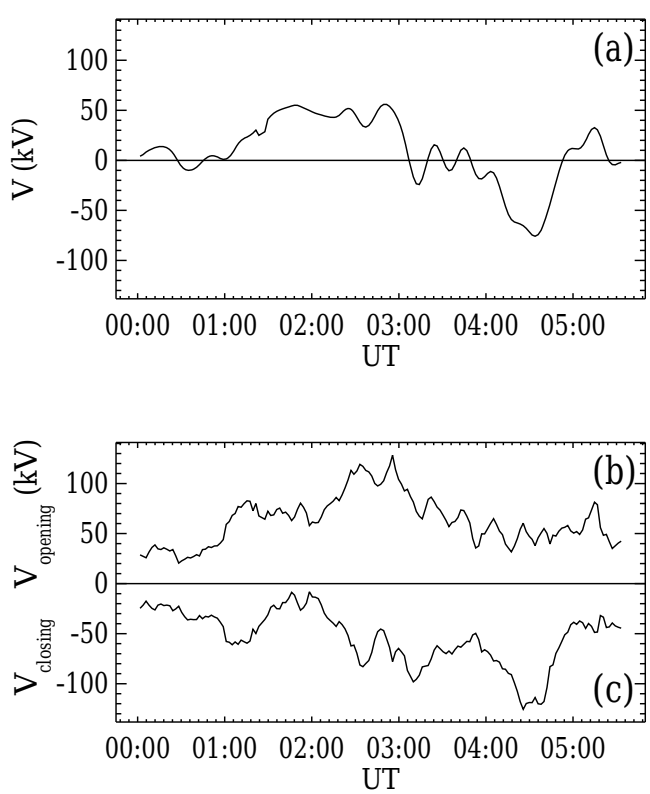

Figure 4. (a) Net reconnection voltage, (b) magnetic flux opening rate and (c) magnetic flux closure rate deduced from SI12 and SuperDARN observations on 3 January 2001. The net voltage in panel (a) is the sum of those in panels (b) and (c).

voltage stabilizes around $60-70 \mathrm{kV}$, a value comparable with the dayside reconnection voltage, allowing a near balance of the magnetic opening and closure rates. The value that we find is significantly larger than the quiet-time reconnection rate of some $\sim 30 \mathrm{kV}$ found by Hubert et al. (2006a), but it remains lower than that found during substorm expansion phases $(\sim 100-140 \mathrm{kV})$, while sawtooth intervals can have reconnection rates significantly above that of substorms, with the open magnetic flux reaching $\sim 1.2 \mathrm{GWb}$ (Hubert et al., 2008).

\subsection{SMC interval on 21 August 2000}

An SMC interval was reported to occur on $21 \mathrm{Au}-$ gust 2000 by De Jong et al. (2007). The AE index remained above $200 \mathrm{nT}$ between 20 August 22:07 UT and $22 \mathrm{Au}$ gust 00:45 UT (with a short-lived excursion below $200 \mathrm{nT}$ on 21 August around 01:22 UT), the AU and AL indices both reaching a high magnitude indicating enhanced magnetospheric activity, as shown in Fig. 5. As for the first interval presented above, the detailed magnetograms from the Canadian CARISMA and Scandinavian IMAGE networks are compatible with the $\mathrm{AE}, \mathrm{AU}$ and $\mathrm{AL}$ indices, as expected. Simultaneous IMAGE FUV and SuperDARN observations were again available on that day, except before 04:32 UT and between 14:10 and 18:30 UT. During more than 1 hour after 04:32 and 18:30 UT, the IMAGE spacecraft was however leaving its perigee and remained too close to the planet for the viewing geometry to allow a global treatment of the images of the proton aurora. No substorm expansion signatures
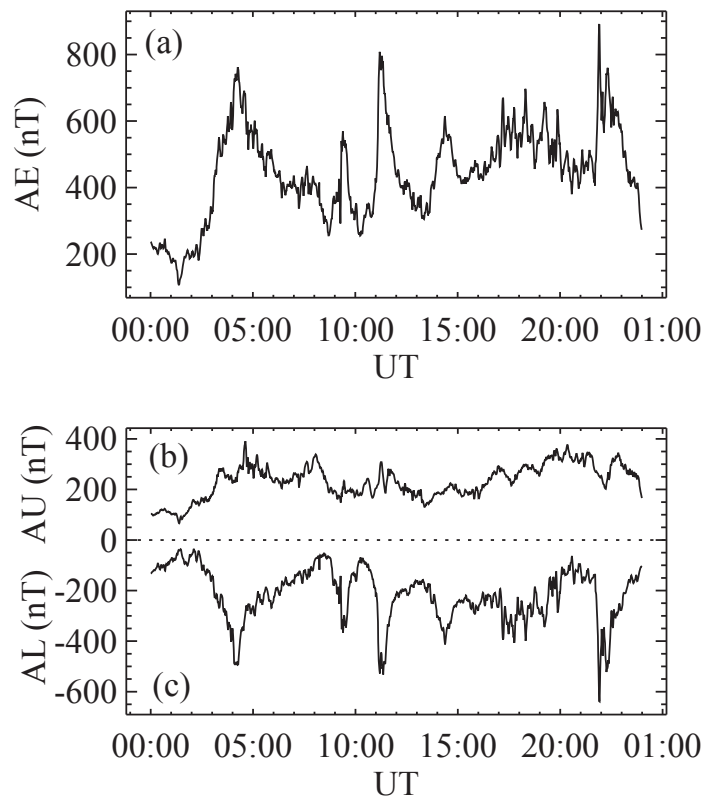

Figure 5. (a) AE, (b) $\mathrm{AU}$ and (c) AL indices recorded on $21 \mathrm{Au}-$ gust 2000 .

appeared in the global FUV imaging of the aurora with the IMAGE-FUV instruments between 04:32 and 09:01 UT. Between 09:01 and 09:30 UT, an auroral bulge appears, indicating that some magnetic flux is being closed in the tail, suggesting that the magnetosphere was not in a state of steady magnetic convection at that time. This holds between 10:50 and 11:50 UT as well, an expansion being observed with a marked poleward retraction of the nightside polar cap developing after 11:04 UT, followed by what seems to be a long recovery phase showing PBIs, i.e. still allowing a small amount of magnetic flux closure. An intensification that we identify as a pseudo-breakup is seen in the FUV auroral images at 21:42 UT, followed by a substorm expansion phase with onset at 21:50 UT, which could signal the end of the steady convection state of the magnetosphere.

Solar wind properties measured by the Wind satellite on 21 August 2001 are shown in Fig. 6. The solar wind velocity and IMF $B_{z}$ component were fairly stable after 02:00 UT. The solar wind density varied a bit more, causing the solar wind dynamic pressure to vary between $\sim 1$ and $2.4 \mathrm{nPa}$ after 02:00 UT. The dynamic pressure remains low despite the rather large density $\left(\sim 8 \mathrm{~cm}^{-3}\right)$, due to the low velocity. The southward orientation of the IMF did allow magnetic reconnection to efficiently open magnetic flux at the Earth's magnetopause during most of the interval, so that active conditions can develop in the magnetosphere, as obviously appears in the AE index shown in Fig. 5a.

The open magnetic flux estimate based on the SI1 2 observations of the proton aurora on 21 August 2000 is shown in Fig. 7. This remains fairly stable, ranging between $\sim 0.8$ and $0.9 \mathrm{GWb}$ from 05:55 to $\sim$ 09:00 UT, as shown by the first 


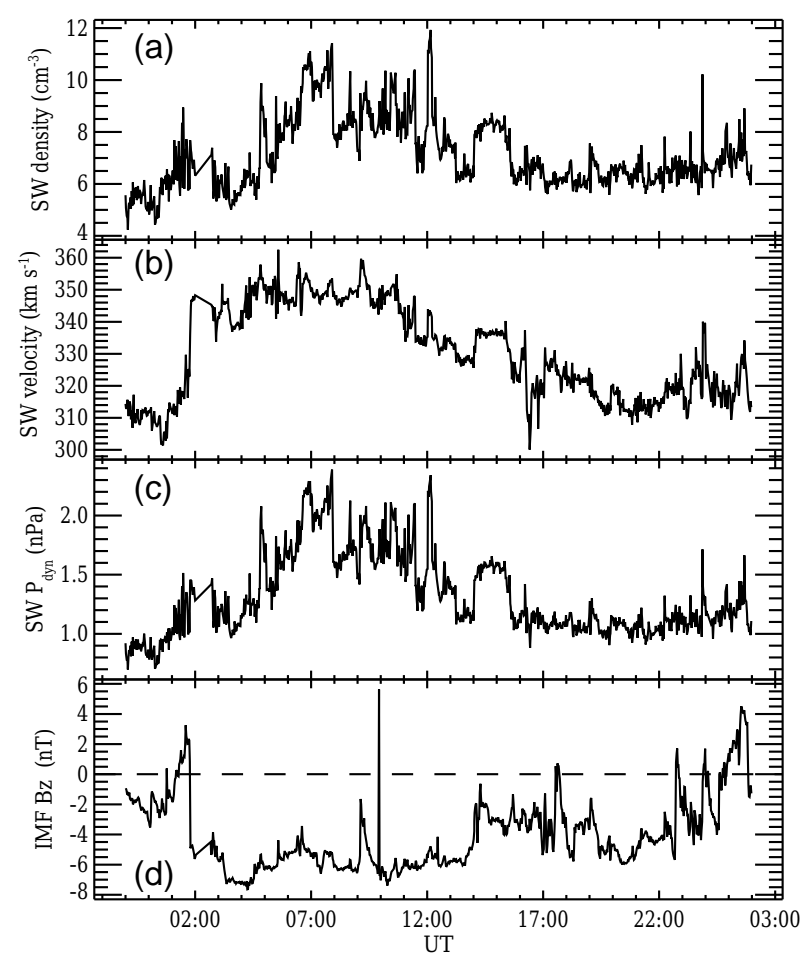

Figure 6. Solar wind properties measured by the Wind satellite on 21 August 2000 in the same format as Fig. 2. The timescale has been shifted by $11 \mathrm{~min}$ to approximately account for the propagation time between the spacecraft and the Earth.

two SI12 images in Fig. 7c. The increased activity seen in the FUV images of the aurora after 09:00 UT is obviously related to the reduction in the magnetic flux starting after 09:00 UT, whereas the activation of the auroral oval observed after 10:50 UT relates to the conspicuous flux closure shown at that time in Fig. 7a. In view of these results, it seems that the magnetosphere slightly departs from a state of SMC between 09:00 and 13:30 UT: the AE index remains compatible with the SMC conditions although it shows transient large variations around 09:30 and 13:00 UT, but significant open flux variations nevertheless take place. There is no variation of the solar wind conditions that could obviously be considered a trigger for the flux closure. The variations of the open magnetic flux deduced from the SI12 proton aurora observations therefore rather appear as transient variations around the more stable conditions of the SMC flow, although our dataset cannot clearly reveal exactly what happened in the magnetosphere at that time. One possibility is the occurrence of several BBFs during a relatively short time interval, since Tanskanen et al. (2005) pointed out that BBFs often occur during SMC intervals, while Hubert et al. (2007) showed that these are related to flux closure. By contrast, the open magnetic flux estimated between 05:55 and 09:00 UT is fully compatible with an SMC state. The open magnetic flux shown in Fig. 7b between 19:45 and $\sim 21: 45$ UT is sta- ble as well, around $\sim 1.02 \mathrm{GWb}$, which is compatible with a state of SMC. Figure 7c shows the SI12 and SuperDARN observations at a few selected UT times between 05:55 and 13:30 UT, with the estimated location of the polar cap boundary overlaid. The high-resolution fluctuations of the boundary that appear in Fig. 7c (and in Fig. 7d, to be discussed later in this paragraph), as in Fig. 3b, are efficiently removed by an appropriate time smoothing. The polar cap area decreases as flux closure is going on, and the brightening of the aurora corresponding to the beginning of the flux closure is obvious around 11:00 UT. The convection pattern is mainly observed on the dayside at this time, such that its stability is hard to assess in sectors where the field lines can reasonably be assumed to map to the nightside lobes of the magnetotail. Figure $7 \mathrm{~d}$ shows the fair stability of the polar cap area prior to 22:00 UT and its decrease when flux closure goes on after 21:42 UT, as shown in Fig. 7b. The ionospheric convection pattern deduced from the SuperDARN observation (averaged on a $5^{\circ}$ MLAT $\times 2 \mathrm{~h}$ MLT grid for the sake of figure readability) shows a fair stability in the 12:00-24:00 MLT sector before 21:48 UT. The convection pattern appears to change during and after the contraction of the polar cap, becoming oriented eastward in the pre-midnight sector. The flux closure that takes place after 21:42 UT (Fig. 7b) that corresponds to a sharp contraction of the polar cap (Fig. 7d) is a clear signature of a substorm expansion, starting with a pseudo-breakup at 21:42 UT and an expansion onset at 21:50 UT (not shown).

The estimated reconnection rates based on the IMAGE SI12 remote sensing of the proton aurora and the SuperDARN observation of the ionospheric convection are shown in Fig. 8. The net reconnection voltage presented in Fig. 8a and $\mathrm{b}$ show, as expected, that the net voltage remains close to zero during intervals of quasi-steady open magnetic flux, when the flux opening and closure rates nearly compensate for each other. This balance seems to be achieved when the dayside reconnection rate, which can be viewed as imposed by the solar wind properties, amounts to $\sim 50 \mathrm{kV}$, prior to 09:00 UT. The expansion phases observed after 09:00 and 10:50 UT obviously result from an intensification of the magnetic flux closure voltage shown in Fig. 8e, the expansion phase observed at 10:50 UT being preceded by an intensification of the magnetic flux opening voltage to $\sim 100 \mathrm{kV}$ (Fig. 8c). This can be seen in the increase in the polar cap area between 10:27 and 11:00 UT (Fig. 7c) followed by its retraction as shown at 11:24 and 12:03 UT. The intensification of the reconnection rate in the magnetotail during the expansion phase observed after 21:42 UT is manifest in Fig. 8f. Prior to that time, the polar cap area delineated at 20:55, 21:01 and 21:22 (Fig. 7d) remains fairly constant, after which it progressively shrinks as shown at 21:48, 22:00 and 22:33 UT. The peak magnitude of the three expansions reported above ranges between 120 and $140 \mathrm{kV}$. Between 19:45 and 21:45 UT, however, the open magnetic flux is quasi-steady, and the net reconnection voltage is close to zero (Fig. 8b). The balance between opening and closure 

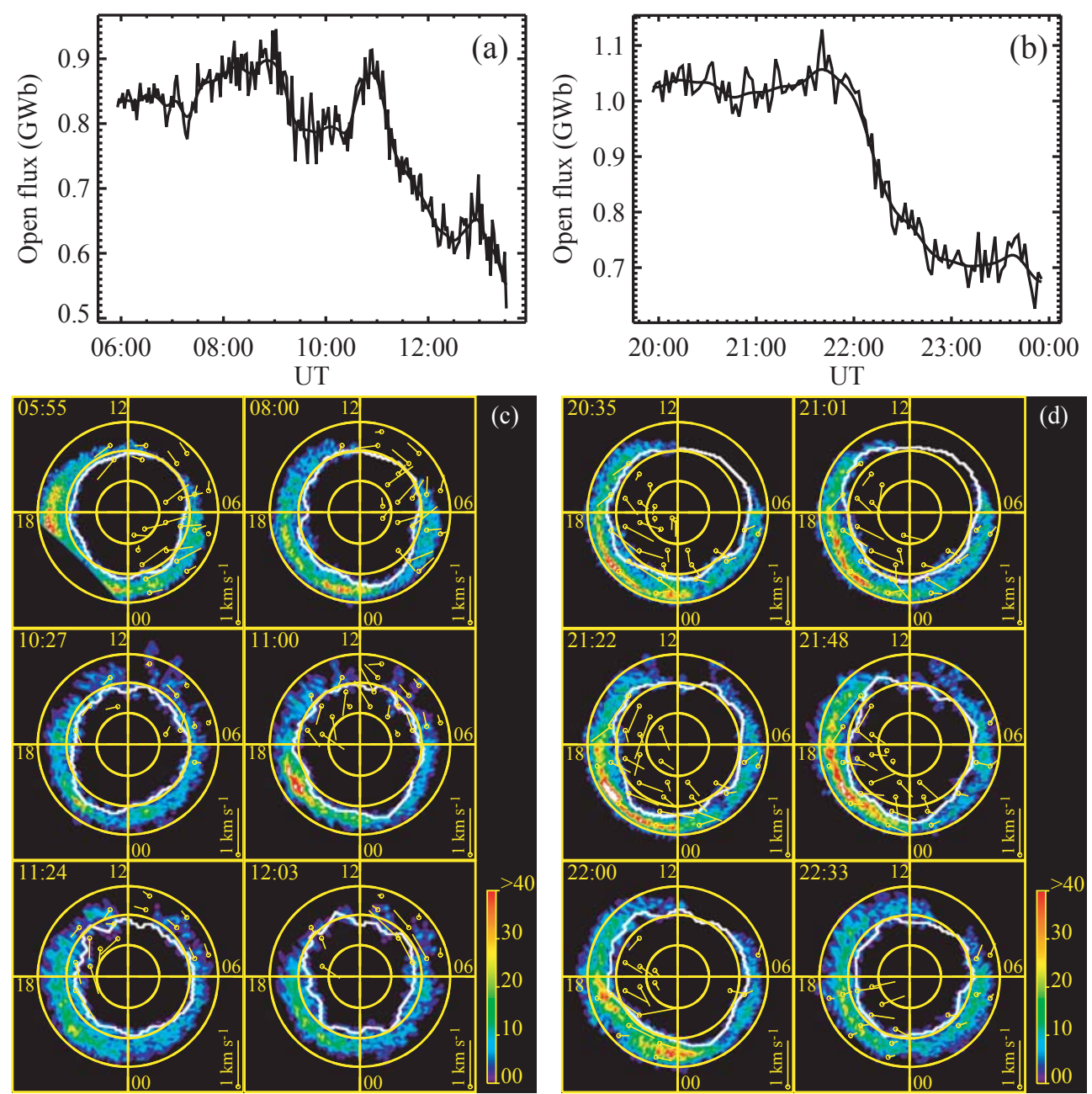

Figure 7. Open magnetic flux estimates using the SI12 images of the proton aurora obtained on 21 August 2000 between (a) 05:55 and 13:30 UT and (b) 19:25 and 23:59 UT, in the same format as Fig. 3a. SI12 images of the proton aurora in geomagnetic polar view (c and d) with the ionospheric convection velocity deduced from SuperDARN observations and with the estimated location of the polar cap boundary, in the same format as Fig. 3b, obtained during the time intervals corresponding to panel (a) and (b), respectively. The average of the SuperDARN measurements of the ionospheric velocity over the polar caps shown is, in chronological order, 392, 467, 212, $332,364,428,493,502,508,453,508$ and $319 \mathrm{~m} \mathrm{~s}^{-1}$.

is achieved when those voltages equilibrate around $\sim 50$ $60 \mathrm{kV}$. The ionospheric convection measured by SuperDARN is also compatible with sustained dayside merging.

\subsection{SMC interval on 26 January 2001}

An SMC interval was reported by De Jong et al. (2007) on 26 January 2001 between 04:00 and 07:50 UT. The AE, AU and $\mathrm{AL}$ indices shown in Fig. 9 reveal that the magnetosphere was active at that time, with AE larger than $200 \mathrm{nT}$ after 04:00 UT, i.e. compatible with SMC conditions. As for the first interval presented above, detailed magnetograms from the Canadian CARISMA and Scandinavian IMAGE networks are compatible with the $\mathrm{AE}, \mathrm{AU}$ and $\mathrm{AL}$ indices, as expected. The solar wind properties measured by the ACE (Advanced Composition Explorer) satellite (Fig. 10) show a stable slow solar wind velocity. The solar wind density varies between $\sim 2$ and $\sim 8 \mathrm{~cm}^{-3}$. The solar wind dynamic pressure remains small throughout the interval. It is rather stable, despite the rapid variation around 06:30 UT, which has a small absolute amplitude. The IMF $B_{z}$ remains steadily southward after 03:00 UT, allowing magnetic reconnection at the magnetopause to open magnetic flux during a prolonged period of time, which can stimulate magnetospheric activity, as expected when AE $>200$ such as during this SMC interval.

The open magnetic flux estimates based on the SI12 remote sensing of the proton aurora are shown in Fig. 11a. After 02:00 UT, the open magnetic flux increases at a slow rate. This growth terminates around 07:30 UT. Indeed, the IMAGE-FUV images of the Earth's aurora show an active oval throughout the interval, with possible brightening such 

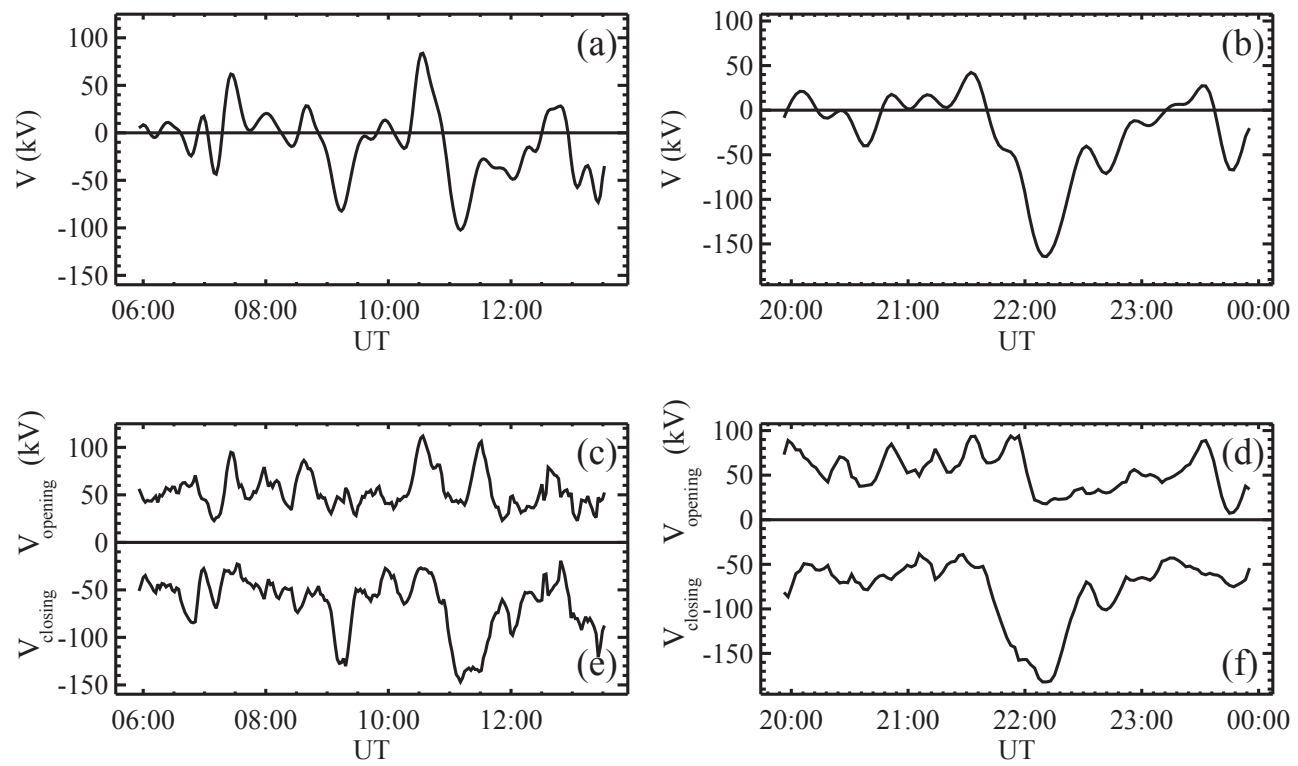

Figure 8. Reconnection voltages estimated using SI12 observations of the proton aurora and SuperDARN measurements of the ionospheric convection on 21 August 2000. The net reconnection voltage (a) and (b) results from the imbalance between the rate of magnetic flux opening (c) and (d) and closure (e) and (f).
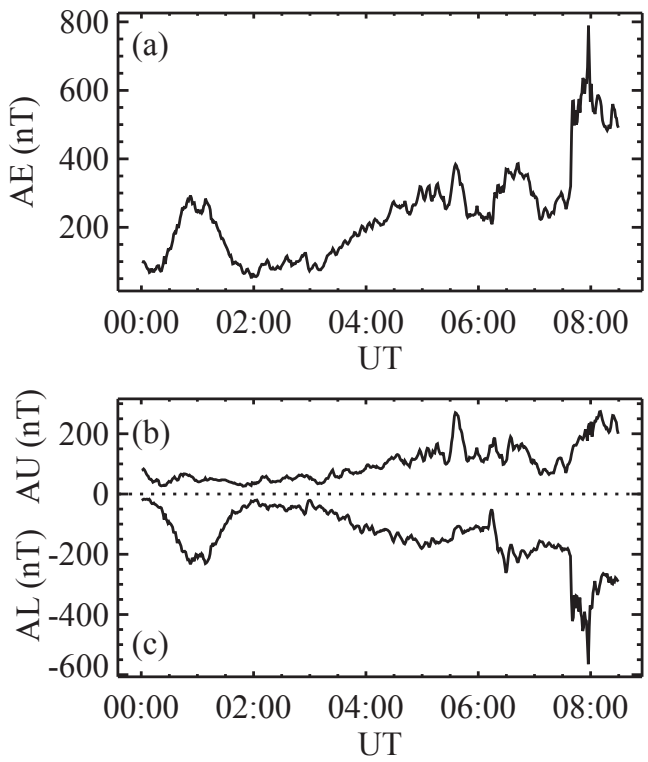

Figure 9. AE (a), AU (b) and AL (c) indices recorded on 26 January 2001. After 04:00 UT, the AE index has a value larger than $200 \mathrm{nT}$, compatible with SMC conditions.

as is observed at 05:00 UT and an expansion onset occurring at 07:30 UT followed by a conspicuous poleward motion of the oval. Consequently, the estimated open magnetic flux decreases rapidly after 07:30 UT. The variation of the polar cap area during this interval can be seen in Fig. 11b that shows the SI12 observation of the proton aurora at several UT times, with the estimated location of the polar cap

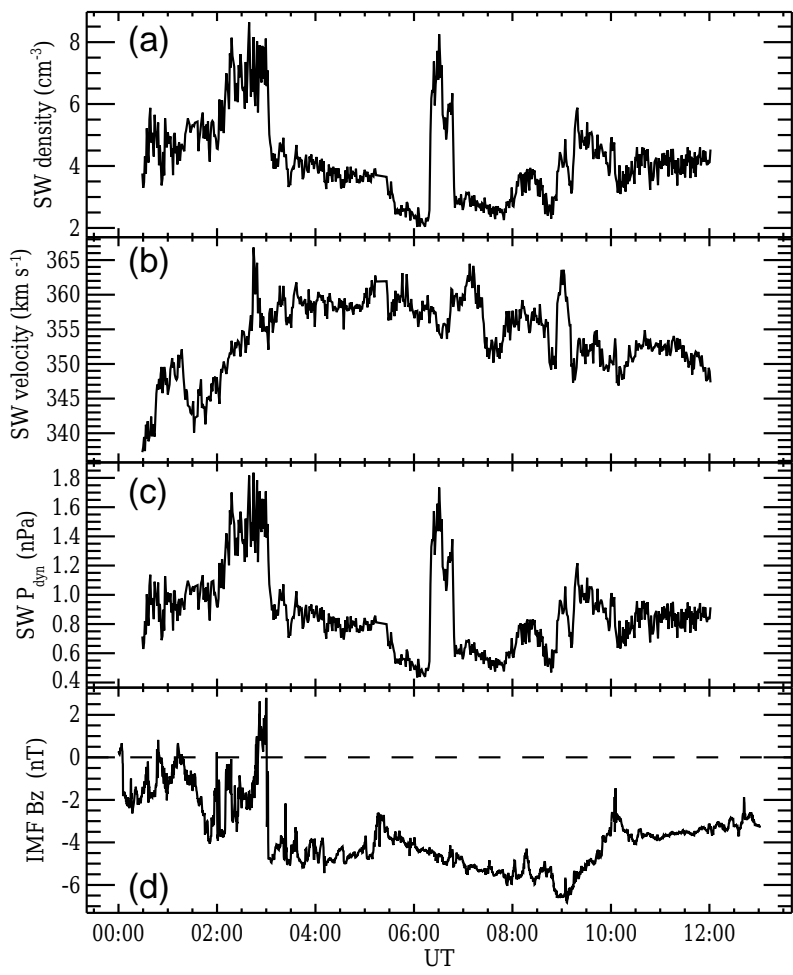

Figure 10. Solar wind properties measured by the ACE satellite on 26 January 2001 (same format as Fig. 2). The timescale has been shifted by $78 \mathrm{~min}$ to approximately account for the propagation time between the spacecraft and the planet. 
boundary: the polar cap area progressively increases between 02:00 and 07:30 UT, after which a dramatic flux closure takes place which reduces the polar cap area (07:32-08:01 UT) as the auroral brightness becomes very large in the pre-midnight sector. Figure $11 \mathrm{~b}$ also shows the ionospheric convection velocity field deduced from the SuperDARN observation, averaged on a $5^{\circ}$ MLAT $\times 2 \mathrm{~h}$ MLT grid for the sake of figure clarity. The gross properties of the convection pattern appear fairly stable versus time, as far as this stability can be analysed from SuperDARN observations during such a long interval. The general convection pattern even seems to persist when flux closure takes place, between 07:32 and 08:00 UT. The expansion is also evident in the net voltage (Fig. 12a) and in the flux closure rate (Fig. 12c) that reached $\sim 165 \mathrm{kV}$. Between 02:00 and 06:00 UT, the closure voltage (Fig. 12b) ranges between $\sim 50$ and $\sim 70 \mathrm{kV}$. The flux closure rate does not exactly compensate for the dayside merging rate, allowing the open magnetic flux to increase slowly as shown in Fig. 11a, b. Nevertheless, we find again the same range for the reconnection voltage, i.e. around $\sim 60 \mathrm{kV}$. This event has, however, the special property of presenting a slowly increasing open magnetic flux, suggesting that we should have some tolerance on the steadiness of the system when considering SMC intervals, i.e. the magnetosphere seems to be capable of developing stable convection, even when a slight regular imbalance persists between flux opening and flux closure, not only producing fluctuations of the open magnetic flux but also allowing a slow but sustained variation of that flux in the long run. This will be considered again on more quantitative grounds in the discussion section. It is interesting to note that, for all three SMC cases that we have detailed, the solar wind velocity was rather low, between $\sim 330$ and $\sim 360 \mathrm{~km} \mathrm{~s}^{-1}$.

\subsection{Other SMC intervals}

We have also analysed several other SMC intervals that occurred on 12 and 30 September, 26 October, 20 November, 22 December 2000, 21 January, 12 May, and 16 November 2001 (De Jong et al., 2007). The general trends of these events are similar to those described above, with some variability. For these events, the solar wind velocity is generally low, below $400 \mathrm{~km} \mathrm{~s}^{-1}$, with one exception on 12 May 2001, for which the solar wind velocity reaches $650 \mathrm{~km} \mathrm{~s}^{-1}$ during a part of the interval but for which the velocity stays around $400 \mathrm{~km} \mathrm{~s}^{-1}$ for most of the interval. The open magnetic flux recorded during these events ranges between $\sim 0.45$ and $0.95 \mathrm{GWb}$, with a general trend around $\sim 0.7 \mathrm{GWb}$. The SMC interval of 9 December 2000 is an exception: the open magnetic flux was between 1 and $1.1 \mathrm{GWb}$ on that day. The opening and closure voltages also have some variability and range between $\sim 40$ and $\sim 80 \mathrm{kV}$. These voltages generally balance around $\sim 50-70 \mathrm{kV}$. The event of 12 May $2001 \mathrm{de}-$ parts from this picture because a very active interval is found between 09:30 and 12:00 UT, with the closure voltage peaking at 150 and $180 \mathrm{kV}$, although the FUV observation of
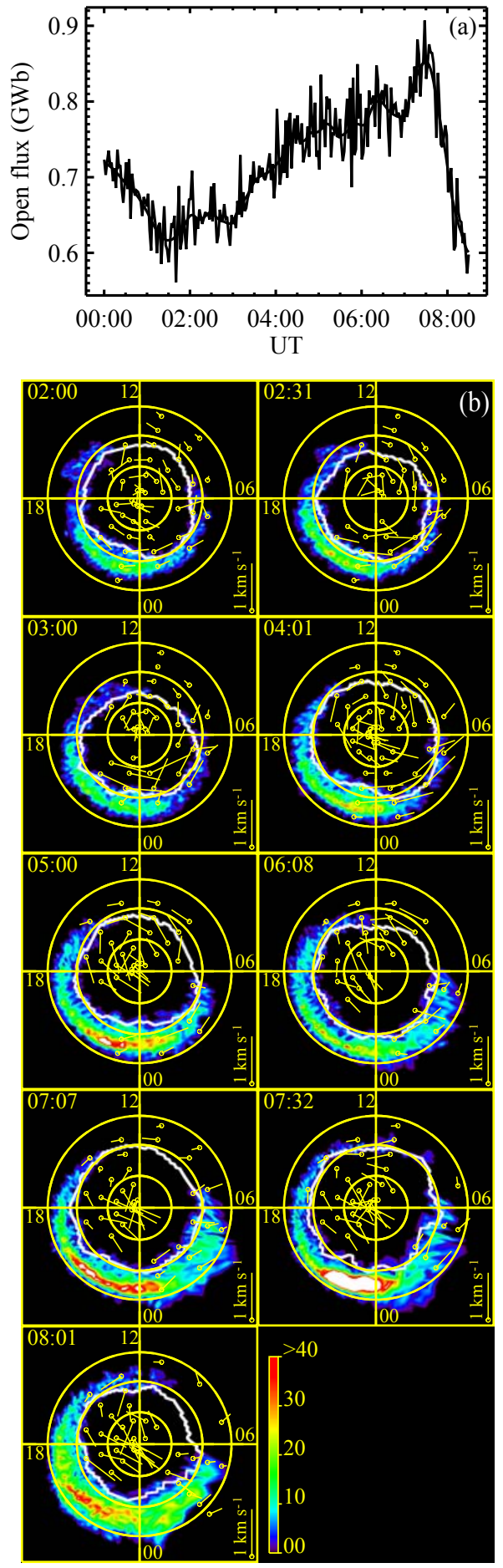

Figure 11. Open magnetic flux estimated using the SI12 images of the Earth's proton aurora on 26 January 2001, in the same format as Figs. 3 and 7. The average of the SuperDARN measurements of the ionospheric velocity over the polar caps shown is, in chronological order, 332, 424, 412,477, 498, 416, 354, 468 and $444 \mathrm{~m} \mathrm{~s}^{-1}$. 

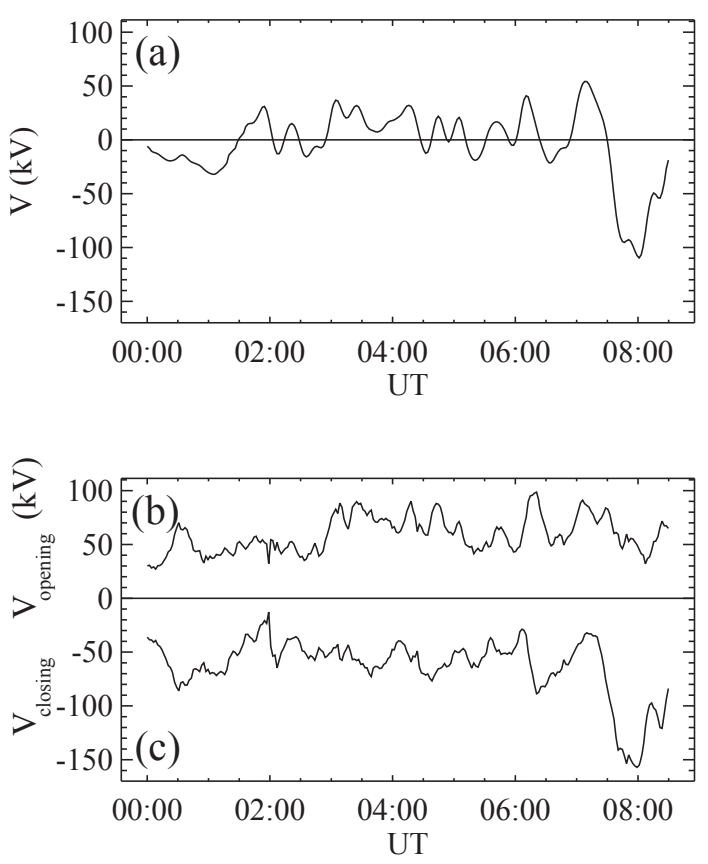

Figure 12. (a) Net reconnection voltage, (b) magnetic flux opening rate and (c) magnetic flux closure rate deduced from SI12 and SuperDARN observations on 26 January 2001. The values in panel (a) are the sum of those of panels (b) and (c).

the aurora does not show a well-defined substorm expansion with an onset but rather a strongly disturbed oval. The opening voltage also reached large values during that part of the interval, with peaks at 150 and $120 \mathrm{kV}$. It must be noted that this disturbed interval is found after the arrival of a moderate dynamic pressure discontinuity in the solar wind and while the solar wind velocity was increasing to values well above $500 \mathrm{~km} \mathrm{~s}^{-1}$. Discontinuities in the solar wind dynamic pressure are known to be capable of stimulating magnetic flux closure in the tail (Boudouridis et al., 2004; Hubert et al., 2006b, 2009). This part of the interval has several properties that strongly depart from those of the other SMC intervals, and it is not clear that it can be considered to be representative of SMC conditions.

\section{Discussion}

\subsection{Properties of SMCs}

We have analysed several intervals of steady magnetospheric convection. Combining the global observations of proton aurora and ionospheric convection for these SMC events, we estimate that the open magnetic flux remains fairly stable between $\sim 0.6$ and $0.9 \mathrm{GWb}$, with some variability from case to case, compatible with the range of open magnetic flux estimated by De Jong et al. (2007) using observations from the WIC instrument. The rates of opening and closure of magnetic flux are typically between $\sim 35$ and $75 \mathrm{kV}$, which roughly corresponds to the previously reported cross-polar cap potential of $\sim 60-90 \mathrm{kV}$ (Sergeev et al., 1996, and references therein), given that the cross-polar cap potential does not necessarily correspond to the reconnection rate in the tail or near the magnetopause. The statistical properties of our estimated open magnetic flux and reconnection rates are summarized in Table 1. The tabulated standard deviations reflect the variability that exists between different SMC intervals but also within a given interval. The net magnetic reconnection voltage reflects the total rate of change in the open magnetic flux. On average, we find it to be close to zero during SMCs (Table 1). However, this quantity is zero on average on long timescales whatever the magnetospheric state, since over sufficiently long intervals the magnetosphere cannot indefinitely accumulate open flux. By contrast, the absolute value of the net reconnection voltage $|V|$ can largely differ from zero on any short timescale, like during the substorm cycle for example. The average of $|V|$ slightly differs from zero during the SMCs studied here and amounts to roughly one third of the estimated individual voltages because the balance between magnetic flux opening and closure can never be perfectly reached (as obviously expected). The slightly positive average value of $V$ could partly result from the slow sustained growth of the open magnetic flux that we record on $26 \mathrm{Jan}-$ uary 2001. This event suggests the possibility for the magnetosphere to show properties typical of steady magnetospheric convection even though the long-term steadiness is not perfectly fulfilled. Nevertheless, the near-stability of the open magnetic flux that we obtain for most of the intervals treated here stands along the same lines as the results found by De Jong and Clauer (2005) and De Jong et al. (2007). We also find indications that the ionospheric convection pattern also remains fairly stable during SMC intervals and that the solar wind velocity was rather low for most of the SMC intervals of our dataset, generally below $\sim 400 \mathrm{~km} \mathrm{~s}^{-1}$. The low solar wind velocity of SMCs was also found in several previous studies (O'Brien et al., 2002; DeJong et al., 2009; Huang et al., 2009; Partamies et al., 2009a, b). It is thought to be related to the lower reconnection electric field of the slower solar wind and with specific internal properties of the magnetosphere possibly regarding magnetotail convection, but a full understanding of the mechanism is still needed.

In an oversimplified model, the magnetosphere could be viewed as composed of two "reservoirs" of magnetic flux: the open and closed magnetic flux reservoirs that exchange magnetic flux through magnetic reconnection taking place at the magnetopause on the dayside and in the plasma sheet on the nightside. This approach allows us to define the residence timescale of the open magnetic flux reservoir as the ratio between its content and its loss rate: $\tau=\Phi / V_{\mathrm{cl}}$. Clearly this expression is exact in the case of a steady state but is employed here more generally as an instantaneous indicator of the timescale on which the open flux reservoir would be emptied in the absence of further open flux production. We computed 
Table 1. Statistical characteristics of the estimated open magnetic flux $(\Phi)$, absolute value of the magnetic flux closure voltage $\left(V_{\mathrm{cl}}\right)$, magnetic flux opening voltage $\left(V_{\mathrm{op}}\right)$, net reconnection voltage $\left(V_{\text {net }}\right)$ and its absolute value $\left(\left|V_{\text {net }}\right|\right)$, residence timescale of the open magnetic flux $(\tau)$, cross-tail plasma velocity estimated assuming a $20 R_{\mathrm{E}}$ tail radius $\left(v_{z} 20\right)$, tail radius estimated from the ACE solar wind data using the model of Petrinec and Russel (1993, 1996) $\left(R_{\mathrm{T}}\right)$, and cross-tail plasma velocity estimated assuming the tail radius is that estimated using the model of Petrinec and Russel $(1993,1996)\left(v_{z}\right)$. The estimated averages and standard deviations are given with the number of SI12 images used in the statistics.

\begin{tabular}{lrrr}
\hline & $m$ & $\sigma$ & $n$ \\
\hline$\Phi(\mathrm{GWb})$ & $0.745 \pm 0.004$ & 0.16 & 1723 \\
$V_{\mathrm{cl}}(\mathrm{kV})$ & $54.6 \pm 0.5$ & 21 & 1723 \\
$V_{\mathrm{op}}(\mathrm{kV})$ & $57.7 \pm 0.5$ & 21 & 1723 \\
$V_{\text {net }}(\mathrm{kV})$ & $3.0 \pm 0.6$ & 27 & 1723 \\
$\left|V_{\text {net }}\right|(\mathrm{kV})$ & $20.1 \pm 0.4$ & 17.6 & 1723 \\
$\tau(\mathrm{h})$ & $4.068 \pm 0.035$ & 1.42 & 1672 \\
$v_{z}\left(\mathrm{~km} \mathrm{~s}^{-1}\right)$ & $9.91 \pm 0.10$ & 4.1 & 1672 \\
$R_{\mathrm{T}}\left(R_{\mathrm{E}}\right)$ & $32.41 \pm 0.13$ & 4.36 & 1159 \\
$v_{z}\left(\mathrm{~km} \mathrm{~s}^{-1}\right)$ & $15.35 \pm 0.16$ & 5.4 & 1159 \\
\hline
\end{tabular}

this ratio for each of the 1723 SMC images of our dataset. We analysed the statistical characteristics of $\tau$ excluding $\sim 3 \%$ of the data with extreme values that we consider as outliers which are able to bias our results (Table 1). We find that $\tau$ ranges across a broad interval of values. It amounts to $4.07 \mathrm{~h}$ on average, with a standard deviation of $1.42 \mathrm{~h}$. The SMC timescale thus appears a bit longer than that of the substorm cycle $(\sim 3 \mathrm{~h})$. Our estimate of $\tau$ is also compatible with the generally accepted timescale of SMCs of 4-6h. The concept of residence timescale can also be used to understand that an SMC can develop despite the apparent unsteadiness of the open magnetic flux on 26 January 2001 (Fig. 11). The timescale $T$ of the growth of the open magnetic flux can be estimated across the SMC interval by comparing the amount of open magnetic flux $\Phi$ with its overall rate of variation as $T=\Phi /(\Delta \Phi / \Delta t)$ with $\Phi \sim(0.62+0.85) / 2=0.735 \mathrm{GWb}$, $\Delta \Phi \sim 0.85-0.62=0.23 \mathrm{GWb}$ and $\Delta t \sim 7 \mathrm{~h}(\Delta Q$ being the variation of quantity $Q$ ), so that $T \sim 19 \mathrm{~h}$, much longer than $\tau$. The concept of steadiness could then be moderated as follows: a significant, sustained, variation of the open magnetic flux (a $30 \%$ increase in this case), which takes place on timescales much longer than the SMC residence timescale of the open magnetic flux, does not necessarily prevent the magnetosphere from satisfying the SMC criteria.

The order of magnitude of the residence time of open flux tubes can also be roughly checked using the convection velocity patterns shown in Figs. 3, 7 and 11. These figures show ionospheric velocity vectors with a magnitude ranging approximately between 0.25 and $0.75 \mathrm{~km} \mathrm{~s}^{-1}$ in the polar cap during several SMC intervals. The detailed trajectory of the ionospheric plasma is hard to determine, but we can estimate that the length of the path across which the plasma threaded by open flux tubes has to travel from the noon sector (roughly mapping to the opening neutral line along the magnetic field lines) to the midnight sector (roughly mapping to the closure neutral line along the magnetic field lines) is on the order of $\sim 4500 \mathrm{~km}$, i.e. the length of a circular arc subtended by an angle of $40^{\circ}$ on a sphere with a radius of $6471 \mathrm{~km}$. We then estimate that the order of magnitude of the residence time ranges between $\sim 1.67$ and $\sim 5 \mathrm{~h}$, compatible with our previous estimate.

For the sake of obtaining orders of magnitude, the transit time can be used to approximately determine the GSM $z$ component of the velocity of the plasma convection in the tail if we consider that $\tau$ roughly represents the time needed for plasma threaded by a particular open flux tube to move from the magnetopause to the reconnection site of the central plasma sheet. Assuming that the plasma has to travel along the distance of the tail radius (in the $z$ direction) arbitrarily set to $20 R_{\mathrm{E}}$ (a reasonable value, after Kivelson and Russel, 1997), the convection velocity $\left(v_{z} 20\right)$ can be estimated for our set of SMC images. These values are statistically summarized in Table 1. The average of $v_{z} 20$ is $9.91 \pm 0.10 \mathrm{~km} \mathrm{~s}^{-1}$ (the standard deviation of the sample being $\sim 4 \mathrm{~km} \mathrm{~s}^{-1}$ ), significantly larger than the average velocity reported by Haaland et al. (2009) based on Cluster data collected between February 2001 and October 2007 (despite an overlap due to the scatter of our dataset); these authors found that the $z$ component of the convection velocity is $\sim 7.7 \mathrm{~km} \mathrm{~s}^{-1}$ on average in the tail lobe, based on Cluster observations gathered between $X_{\mathrm{GSM}} \sim-5$ and $X_{\mathrm{GSM}} \sim-21 R_{\mathrm{E}}$. This suggests that the enhanced activity of SMC intervals has a convection velocity enhanced by $\sim 30 \%$ as counterpart in the tail, SMC conditions representing only a subset of all the conditions possibly included in the study of Haaland et al. (2009). The value of the tail radius $R_{\mathrm{T}}$ can also be estimated based on solar wind data from the ACE satellite, using the model of Petrinec and Russel $(1993,1996)$, which relates the tail radius to the solar wind dynamic pressure and IMF $B_{z}$. This value was computed at the time of each SMC image of our SMC dataset when suitable (time-shifted) ACE data were available. We find that the tail radius was $R_{\mathrm{T}} \sim 32 R_{\mathrm{E}}$ on average at far downtail distance (Table 1). We estimated that the tail radius $R_{\mathrm{T}}$ reaches $90 \%$ of its asymptotic value at a distance of $\sim 36 R_{\mathrm{E}}$ downtail from the Earth on average. Computing again the order of magnitude of the $z$ component of the convection velocity in the tail using the estimated $R_{\mathrm{T}}$ (for every SMC image), we find $v_{z} \sim 15.35 \pm 0.16 \mathrm{~km} \mathrm{~s}^{-1}$ on average (Table 1). This value is essentially twice as large as that reported by Haaland et al. (2009). Our estimate can however be viewed as an upper bound to $v_{z}$ because the asymptotic, far tail radius is the upper bound to the distance plasma has to travel along the $z$ direction before reaching the reconnection site in the central plasma sheet. It nevertheless remains that we infer an SMC convection velocity (in the tail) apparently larger than the global average value. The larger 
Table 2. Typical values of the open magnetic flux and magnetic reconnection rates estimated during four magnetospheric modes (pk stands for peak values).

\begin{tabular}{lrrrr}
\hline & Quiet time & SMC & Substorm & Sawtooth \\
\hline$\Phi(\mathrm{GWb})$ & $<\sim 0.4-0.5$ & $\sim 0.6-0.9$ & $\sim 0.65-0.9$ & $\sim 1.0-1.4$ \\
& & & $0.7-0.96 \mathrm{pk}$ & \\
$V_{\mathrm{op}}, V_{\mathrm{cl}}(\mathrm{kV})$ & $<\sim 40$ & $\sim 35-75$ & $\sim 70-140 \mathrm{pk}$ & $\sim 60-160$ \\
\hline
\end{tabular}

convection velocity that we infer here does not stem from an exceptionally large rate of reconnection in the tail. Simply, the solar wind velocity is particularly low during most of the SMC intervals, giving a very low solar wind dynamic pressure used in the Petrinec and Russel $(1993,1996)$ model, which then produces a rather large estimate for the tail radius and a proportionally large value for $v_{z}$. Given that $\tau \sim 4.1 \mathrm{~h}$, one would retrieve $v_{z} \sim 7.7 \mathrm{~km} \mathrm{~s}^{-1}$ if the plasma had to travel across a distance of $\sim 18 R_{\mathrm{E}}$, only a little more than the half of the average asymptotic tail radius that we have estimated. It must be noted that, in the frozen-in approximation, an enhanced $v_{z}$ leads to an enhanced cross-tail electric field $E_{y}$, compatible with a sustained magnetic flux closure rate, which is one of the defining features of SMC intervals.

\subsection{Intercomparison of magnetospheric convection modes}

The statistical properties that we infer for SMC intervals should be placed in the context of the previous studies of $\mathrm{Hu}-$ bert et al. (2006a, 2008) concerning quiet-time conditions, the substorm cycle and sawtooth events. We propose that the magnetosphere can exhibit four different modes differing from each other through the amount of open magnetic flux in the magnetosphere and the magnetic reconnection rates. Table 2 lists the typical values found for these four modes, namely (in ascending reconnection rate) quiet-time conditions, SMC, the substorm cycle and sawtooth modes. For the substorm mode, we analysed an extended set of 629 substorms found between May 2000 and November 2001. We used the substorm list of Frey et al. (2004) and examined every interval individually to verify that the estimated magnetic fluxes and reconnection voltages were suitable: we excluded intervals which could apparently not really be considered a substorm, such as shock-induced auroral activity or parts of sawtooth event intervals for example. More importantly, we excluded intervals with insufficient data coverage, or for which our automatic software did not properly treat the data. We were left with 629 intervals for which the average value of the peak value of the open magnetic flux is $\sim 0.83 \mathrm{GWb}$ with a standard deviation of $0.13 \mathrm{GWb}$ and peak reconnection voltages of $103 \mathrm{kV}$ (closure) and $104 \mathrm{kV}$ (opening) on average, both with a standard deviation of $\sim 36 \mathrm{kV}$ so that, on average over the studied set of substorms, the average of the peak values of the opening and closure voltages can be con- sidered statistically equal. The substorm growth and expansion phases can be expected to have different properties considering that the magnetosphere accumulates open magnetic flux during the growth phase, which is mostly closed somewhat later during the expansion phase. Focusing on the expansion phase, the mean value of the flux and closure reconnection voltage are computed over each individual interval, then the average and standard deviation of this set of mean values is computed. We find the averages and standard deviations (not to be confused with the uncertainty over the estimated averages) $\langle\Phi\rangle=0.7743 \mathrm{GWb}, \sigma_{\Phi}=0.1214 \mathrm{GWb}$ and $\left\langle V_{\mathrm{cl}}\right\rangle=-66.70 \mathrm{kV} \sigma_{V_{\mathrm{cl}}}=22.05 \mathrm{kV}$, while the opening reconnection voltage is more relevantly appreciated during the growth phase: $\left\langle V_{\mathrm{op}}\right\rangle=64.36 \mathrm{kV} \sigma_{\mathrm{Vop}}=28.93 \mathrm{kV}$. Table 2 instead lists the average peak values for the substorm mode because we think that the explosive nature of the substorm expansion phase is better described by extrema than by averages, although averages remain valuable indicators.

The sawtooth numbers listed in Table 2 are based on the seven intervals treated by Hubert et al. (2008), rejecting the case on 4 October 2000, which had a somewhat higher noise level due to a poorer determination of the open/closed boundary location. For the open magnetic flux, Table 2 gives the average plus/minus the standard deviation of the main local maxima reached during the set of sawtooth events $\left(<\Phi_{\max }>=1.21 \mathrm{GWb}, \sigma_{\Phi \max }=0.21 \mathrm{GWb}\right)$. Indeed, several intervals of flux accumulation and flux closure follow each other during a sawtooth event. The average flux closure voltage and its standard deviation are obtained by isolating the most active subintervals of each sawtooth interval of Hubert et al. (2008). We selected the $66 \%$ data points with the largest reconnection rates of each interval (separately for opening and closure), grouped those subsets together and computed the value of the average and the standard deviation of that subset $\left(\left|<V_{\mathrm{cl}}>\right|=110 \mathrm{kV}, \sigma_{\mathrm{Vcl}}=49 \mathrm{kV}\right.$, $<V_{\mathrm{op}}>=109 \mathrm{kV}, \sigma_{\mathrm{Vop}}=45 \mathrm{kV}$ ) from which we obtain the range listed in Table 2. (If thresholding is ignored, we find $\left\langle V_{\mathrm{op}}>=85 \mathrm{kV}, \sigma_{\mathrm{Vop}}=52 \mathrm{kV}\right.$ and $\left|<V_{\mathrm{cl}}>\right|=86 \mathrm{kV}$, $\sigma_{V_{\mathrm{cl}}}=50 \mathrm{kV}$.) The thresholding procedure that we use is somewhat arbitrary. We simply inspected the flux closure time series and saw that this $66 \%$ thresholding excludes most of the less active subintervals, during which the flux closure rate is reduced while flux opening already announces the following subinterval of intense flux closure. We also noticed that the same threshold efficiently rejects intervals with a somewhat reduced flux opening rate when applied to the flux opening reconnection voltage. Figure 13 shows the application of the thresholding to the sawtooth event that occurred on 24 October 2002 detailed by Hubert et al. (2008). The sawtooth numbers listed in Table 2 must thus be considered indicative of the main properties of sawtooth events rather than rigidly established values. It is also surprising that on average, $V_{\mathrm{cl}}$ and $V_{\mathrm{op}}$ are roughly equal, no matter whether thresholding is included or not. One would expect that, in the long run, the amount of magnetic flux that is 


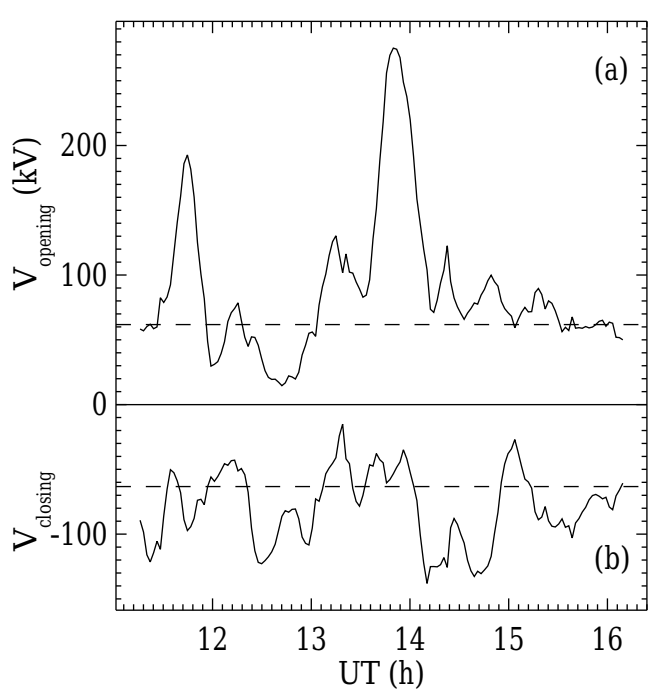

Figure 13. Dayside (a) and nightside (b) reconnection voltages determined by Hubert et al. (2008) during the sawtooth event that occurred on 24 October 2002. The horizontal dashed lines show how the $66 \%$ thresholding separates large from smaller reconnection rates.

opened on the dayside is eventually closed in the tail, so that the time-integrated voltages should become equal. Having the same voltage values also suggests in addition that, on average, flux opening and flux closure take place during time intervals of roughly the same duration. This property still needs an explanation, which is probably to be found in the details of the solar-wind-driven magnetohydrodynamics (MHD) plasma flow inside of the magnetosphere. We speculate that this property is a general characteristic of the magnetosphere rather than of the sawtooth mode because the correspondence between average opening and closure voltages is also found within a few kilovolts for the substorm mode. Indeed, this correspondence is even found for the peak reconnection voltages, with averages listed in Table 2 .

Despite what Table 2 may suggest, there exists some overlap between the four listed modes, as is represented in Fig. 14, which sorts them by increasing open flux and reconnection rate. The behaviour of such a complex coupled system comprising the solar wind, the magnetosphere and the ionosphere can obviously not be reduced to just a few numbers, even though the amount of open magnetic flux and the reconnection rates are among the most important ones. This mode classification should not hide the key role of the solar wind properties in the dynamics of the magnetosphere, as has been statistically studied by DeJong et al. (2009), who highlighted the importance of the IMF $B_{z}$ component and of the solar wind velocity, temperature and Mach number (which control the dayside reconnection rate in large measure). For example, the importance of having a low solar wind velocity for the development of the SMC mode does not appear in the present analysis. One could speculate that the SMC mode
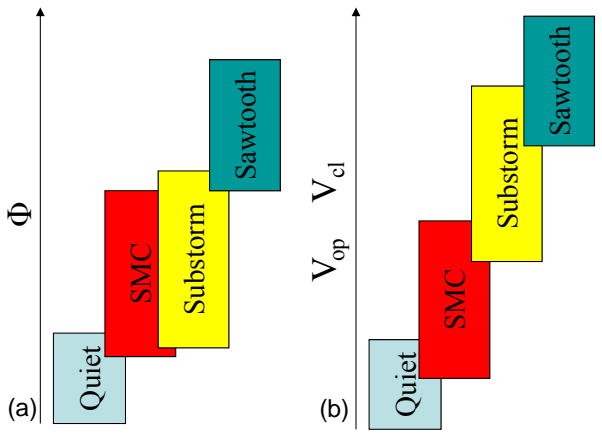

Figure 14. Different magnetospheric modes organized versus the open magnetic flux $\Phi$ (left) and versus the opening and closure voltages $V_{\mathrm{op}}$ and $V_{\mathrm{cl}}$ (right).

has to be tuned such that the rate of transport of magnetic flux from the magnetopause to the plasma sheet inside of the magnetosphere matches the rate at which new open flux tubes are transported in the nearby interplanetary medium as the solar wind flows by the Earth, keeping the magnetic topology sufficiently stable to avoid the breakup of an expansion phase. If the rate of transport inside of the tail is limited by the internal properties of the magnetosphere, then it would seem natural that the solar wind velocity has to stay within some acceptable bounds (to be determined) and thus stays relatively small. Further detailed analysis would however be needed to assess the validity of that idea.

The magnetospheric modes considered in this study are driven by the solar wind input: the quiet-time mode is the consequence of a reduced merging rate at the dayside magnetopause when the IMF is oriented (approximately) northward, the substorm cycle develops based on the imbalance on a short timescale (less than $\sim 3 \mathrm{~h}$ ) between magnetic flux closure and opening, and the SMC condition can develop when the dayside reconnection rate ranges around $55 \pm 20 \mathrm{kV}$ (Table 1) (at low solar wind velocity), while the sawtooth event stems from the overfeeding of the magnetosphere with open magnetic flux by very intense magnetic reconnection on the dayside (Hubert et al., 2006a, 2008). The four magnetospheric modes presented here thus reflect the existence of (at least) four different particular modes of interaction between the magnetosphere and the interplanetary medium, at the heart of which the process of magnetic reconnection plays a key role.

The value of the dayside reconnection rate is not the only important parameter. The timing is a factor as well, as illustrated by Partamies et al. (2009a): the substorm develops after a growth phase with an intense reconnection, whereas the sawtooth and SMC events have an intense dayside merging rate throughout the time interval. In our mode classification, the average open flux is only slightly larger during substorms than during SMC intervals, but the peak values met during substorms are found to be nearly $0.1 \mathrm{GWb}$ larger than the SMC value, which is in line with the results found by Huang 
et al. (2009). The mode classification versus reconnection voltage reflects how the intensity of the coupling between the solar wind and the magnetospheric environment drives the response of the coupled magnetosphere-ionosphere system. Our results are in line with the lines of those found by Partamies et al. (2009b), who expressed this interaction in terms of the $\varepsilon$ coupling function and the solar wind electric field. It also appears that the reconnection voltage better discriminates between the different modes than the open magnetic flux, which presents a more pronounced overlap between modes. This indicates that the key that separates the various magnetospheric modes presented in this study is to be found in the response of the magnetosphere to the coupling with the solar wind, and future studies should be conducted to better identify the solar wind parameters that drive the magnetospheric response. Three questions that we think need to be answered are as follows.

- Does the dayside reconnection rate history fully determine the magnetospheric mode?

- Does magnetospheric preconditioning influence the development of the different magnetospheric modes (along the lines of the results of Juusola et al., 2013, and Kissinger et al., 2012a, b)?

- Do the properties of the magnetospheric modes depend on season?

\section{Conclusions}

Our study shows that SMC intervals can develop when the reconnection rates of the dayside magnetopause and of the magnetotail roughly match at values around $\sim 55 \pm 20 \mathrm{kV}$. The open magnetic flux then remains between $\sim 0.6$ and $0.9 \mathrm{GWb}$. These SMC values depart from those occurring during quiet times, the substorm cycle and sawtooth events. Therefore, we classify these four phenomena as four magnetospheric modes having different open magnetic flux and reconnection rates, as summarized in Fig. 14. We estimate the order of magnitude of the residence timescale of open magnetic flux to be $\sim 4 \mathrm{~h}$ during SMC intervals, longer than the classical timescale of the substorm cycle, which further justifies considering both as distinct, full magnetospheric modes. We also notice that SMC conditions are more likely to develop when the solar wind velocity is low, but the physical reason for that condition still escapes full understanding.
Data availability. The IMAGE-FUV data can be obtained from the CDAWeb service of the Goddard Space Flight Center (https: //cdaweb.gsfc.nasa.gov/sp_phys/; Viljanen and Häkkinen, 1997; Mende et al., 2000a, b). The SuperDARN data can be obtained on request from http://superdarn.jhuapl.edu/ (Ruohoniemi et al., 1987). The magnetometer data from the Scandinavian IMAGE network are available online at http://space.fmi.fi/image/beta/ (Viljanen and Häkkinen, 1997; Mann et al., 2008), and those from the CARISMA Canadian network can be found at http://www.carisma.ca/ (Mann et al., 2008). The solar wind data from the ACE (Stone et al., 1998) and Wind (Acuña et al., 1995) satellites are available from the CDAWeb service (https://cdaweb.gsfc.nasa.gov/sp_phys/). The $\mathrm{AE}, \mathrm{AU}$ and AL indices can be found at the World Data Center for Geomagnetism in Kyoto (http://wdc.kugi.kyoto-u.ac.jp/aedir/, Davis and Sugiura, 1966). 


\section{Appendix A: Computation of the open flux threading the polar cap}

The polar cap boundary is a curve traced at the surface of a sphere. Using polar and cylindrical coordinates thus appear as natural choices (Fig. A1). The polar cap boundary can be represented as a Fourier series giving $\rho$, the length of the projection of vector $\boldsymbol{r}$ (joining the Earth center to any point of the boundary) in the equatorial plane expressed as a function of the azimuth angle $\varphi$. We note that, for every value of $\varphi$, the polar angle $\theta$ will vary across the polar cap from 0 to a value $\theta_{\max }(\varphi)$ given by $\arcsin (\rho(\varphi) / r)$. The magnetic flux threading the polar cap will be the integral across the polar cap of the dot product between the magnetic induction field and the vector normal to the surface, i.e. the radial component $B_{\mathrm{r}}$ of the magnetic induction field:

$$
\Phi=\int_{0}^{2 \pi} \mathrm{d} \varphi \int_{0}^{\arcsin (\rho(\varphi) / r)} \mathrm{d} \theta r^{2} \sin (\theta) B_{\mathrm{r}} .
$$

For a dipole field of magnetic moment $M$, the radial component of the field is

$$
B_{\mathrm{r}}=\frac{-\mu_{0} M}{2 \pi} \frac{\cos (\theta)}{r^{3}}
$$

so the integral (Eq. A1) becomes

$$
\begin{aligned}
\Phi & =r^{2} \int_{0}^{2 \pi} \mathrm{d} \varphi \int_{0}^{\arcsin \left(\frac{\rho(\varphi)}{r}\right)} \mathrm{d} \theta \frac{-\mu_{0} M}{2 \pi} \frac{\cos (\theta)}{r^{3}} \sin (\theta) \\
& =\frac{-\mu_{0} M}{2 \pi r} \int_{0}^{2 \pi} \mathrm{d} \varphi\left[\frac{\sin ^{2}(\theta)}{2}\right]_{0}^{\arcsin \left(\frac{\rho(\varphi)}{r}\right)} \\
& =\frac{-\mu_{0} M}{4 \pi r} \int_{0}^{2 \pi} \mathrm{d} \varphi\left(\frac{\rho(\varphi)}{r}\right)^{2} .
\end{aligned}
$$

Now, the ratio $\rho^{*}=\rho(\varphi) / r$ can be represented using the Fourier series:

$\rho^{*}=\frac{\rho(\varphi)}{r}=c+\sum_{k=1}^{n} a_{k} \sin (k \varphi)+\sum_{k=1}^{n} b_{k} \cos (k \varphi)$.
Its square is

$$
\begin{aligned}
\rho^{* 2} & =c^{2}+\sum_{k=1}^{n} \sum_{j=1}^{n} a_{k} a_{j} \sin (k \varphi) \sin (j \varphi) \\
& +\sum_{k=1}^{n} \sum_{j=1}^{n} b_{k} b_{j} \cos (k \varphi) \cos (j \varphi) \\
& +2 c \sum_{k=1}^{n} a_{k} \sin (k \varphi)+2 c \sum_{k=1}^{n} b_{k} \cos (k \varphi) \\
& +2 \sum_{k=1}^{n} \sum_{j=1}^{n} a_{k} b_{j} \sin (k \varphi) \cos (j \varphi)
\end{aligned}
$$

so that the integral of $\rho^{* 2}$ for $\varphi$ varying between $\varphi_{0}$ and $\varphi_{1}$ is

$$
\begin{aligned}
& \int_{\varphi_{0}}^{\varphi_{1}} \mathrm{~d} \varphi \rho^{* 2}=c^{2}\left(\varphi_{1}-\varphi_{0}\right) \\
& +\sum_{k=1}^{n} \sum_{\substack{j=1 \\
j \neq k}}^{n} a_{k} a_{j}\left[\frac{k \cos (k \varphi) \sin (j \varphi)-j \cos (j \varphi) \sin (k \varphi)}{j^{2}-k^{2}}\right]_{\varphi_{0}}^{\varphi_{1}} \\
& +\sum_{k=1}^{n} \sum_{\substack{j=1 \\
j \neq k}}^{n} b_{k} b_{j}\left[\frac{j \cos (k \varphi) \sin (j \varphi)-k \cos (j \varphi) \sin (k \varphi)}{j^{2}-k^{2}}\right]_{\varphi_{0}}^{\varphi_{1}} \\
& -2 c \sum_{k=1}^{n} \frac{a_{k}}{k}[\cos (k \varphi)]_{\varphi_{0}}^{\varphi_{1}}+2 c \sum_{k=1}^{n} \frac{b_{k}}{k}[\sin (k \varphi)]_{\varphi_{0}}^{\varphi_{1}} \\
& +2 \sum_{k=1}^{n} \sum_{\substack{j=1 \\
j \neq k}}^{n} a_{k} b_{j}\left[\frac{k \cos (k \varphi) \cos (j \varphi)-j \sin (j \varphi) \sin (k \varphi)}{j^{2}-k^{2}}\right]_{\varphi_{0}}^{\varphi_{1}} \\
& +\sum_{k=1}^{n} a_{k}^{2}\left[\frac{\varphi}{2}-\frac{\sin (2 k \varphi)}{4 k}\right]_{\varphi_{0}}^{\varphi_{1}}+\sum_{k=1}^{n} b_{k}^{2}\left[\frac{\varphi}{2}+\frac{\sin (2 k \varphi)}{4 k}\right]_{\varphi_{0}}^{\varphi_{1}} \\
& -2 \sum_{k=1}^{n} a_{k} b_{k}\left[\frac{\cos ^{2}(k \varphi)}{2 k}\right]_{\varphi_{0}}^{\varphi_{1}} \text {. }
\end{aligned}
$$

Using expression Eq. (A6) in Eq. (A3) and letting $\varphi_{0}=0$ and $\varphi_{1}=2 \pi$, we obtain

$$
\Phi=\frac{-\mu_{0} M}{4 \pi r} 2 \pi\left(c^{2}+\sum_{k=1}^{n} \frac{a_{k}^{2}}{2}+\sum_{k=1}^{n} \frac{b_{k}^{2}}{2}\right) .
$$

An appropriate sign convention can be chosen to make $\Phi$ a positive number. This is equivalent to choosing the downward orientation of the normal vector in the north polar cap when computing the open magnetic flux. The time derivative of $\Phi$ is an electric voltage in application of Faraday's law: it is the net reconnection voltage. This voltage relates to the electric field measured along the moving open/closed field line boundary taken at any altitude (Siscoe and Huang, 1985; see also Grocott et al., 2002; Milan et al., 2003, 2004; Milan, 2004; Hubert et al., 2006a, and references therein): the circulation of the electric field along a closed loop is the 


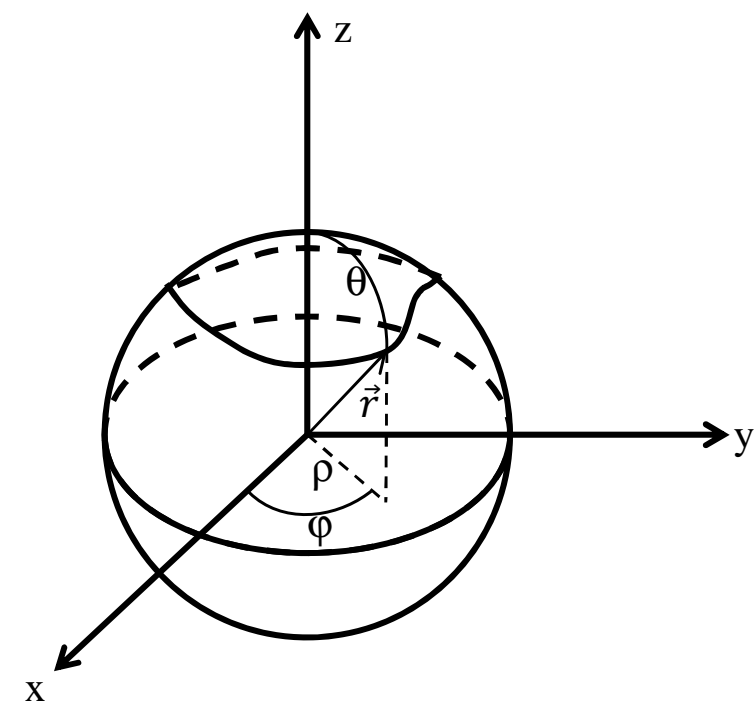

Figure A1. Geometry of a polar cap delineated using a Fourier series, represented in polar coordinates.

electric voltage that corresponds to the rate of change in the magnetic flux through any surface delineated by that closed curve and in particular in the open magnetic flux threading the polar cap delineated by the moving open/closed field line boundary detected at ionospheric altitude. This electric field has two contributions: the ionospheric field and the motional field that accounts for the motion of the boundary. If the magnetic field lines can be considered electric equipotential curves (which cannot always be strictly true) then one can consider that positive contributions to the circulation integral represent an increase in the open magnetic flux and thus refer to reconnection at (or near) the magnetopause, while negative contributions rather refer to flux closure in the magnetotail (providing that a consistent travel direction has been chosen to perform the circulation integral). 
Competing interests. The authors declare that they have no conflict of interest.

Acknowledgements. Benoît Hubert is supported by the Belgian Fund for Scientific Research (FRS). This work was funded by the PRODEX program of the European Space Agency (ESA). Work at Leicester was supported by STFC grant ST/N00749/1. ACE level 2 data were provided by N. F. Ness (MFI) and D. J. McComas (SWEPAM) and the ACE Science Centre. Data from the CARISMA magnetometer network were made available online thanks to Ian Mann. We also thank the institutes which maintain the IMAGE magnetometer array and make the magnetograms available online. We thank the NASA-IMAGE team and in particular the IMAGE-FUV team.

The topical editor, E. Roussos, thanks A. Dejong and one anonymous referee for help in evaluating this paper.

\section{References}

Acuña, M. H., Ogilvie, K. W., Baker, D. N., Curtis, S. A., Fairfield, D. H., and Mish, W. H.: The Global Geospace Science Program and Its Investigations, Space Sci. Rev., 71, 521, doi:10.1007/BF00751323, 1995 (data available at: https:// cdaweb.gsfc.nasa.gov/sp_phys/).

Akasofu, S. I.: The development of the auroral substorm, Planet. Space Sci. 12, 273-282, 1964.

Boakes, P. D., Milan, S. E., Abel, G. A., Freeman, M. P., Chisham, G., and Hubert, B.: A statistical study of the open magnetic flux content of the magnetosphere at the time of substorm onset, Geophys. Res. Lett., 36, L04105, doi:10.1029/2008GL037059, 2009.

Boudouridis, A., Zesta, E., Lyons, L. R., Anderson, P. C., and Lummerzheim, D.: Magnetospheric reconnection driven by solar wind pressure fronts, Ann. Geophys., 22, 1367-1378, doi:10.5194/angeo-22-1367-2004, 2004.

Cowley, S. W. H. and Lockwood, M.: Excitation and decay of solar wind-driven flows in the magnetospere-ionosphere system, Ann. Geophys., 10, 103-115, 1992.

Davis, T. N. and Sugiura, M.: Auroral activity index AE, and its universal time variations, J. Geophys. Res., 71, 3, 785-801, doi:10.1029/JZ071i003p00785, 1966 (data available at: http:// wdc.kugi.kyoto-u.ac.jp/aedir/).

DeJong, A. D. and Clauer, C. R.: Polar UVI Images to study steady magnetospheric convection events: initial results, Geophys. Res. Lett., 32, L24101, doi:10.1029/2005GL024498, 2005.

DeJong, A. D., Cai, X., Clauer, R. C., and Spann, J. F.: Aurora and open magnetic flux during isolated substorms, sawteeth, and SMC events, Ann. Geophys., 25, 1865-1876, doi:10.5194/angeo-25-1865-2007, 2007.

DeJong, A. D., Rideley, A. J., Cai, X., and Clauer, C. R.: A statistical study of BRIs (SMCs), isolated substorms, and individual sawtooth injections, J. Geophys. Res., 114, A08215, doi:10.1029/2008JA013870, 2009.

Dungey, J. W.: Interplanetary field and the auroral zones, Phys. Res. Lett. 6, 47-48, 1961.

Dungey, J. W.: The length of the magnetospheric tail, J. Geophys. Res., 70, 1753, doi:10.1029/JZ070i007p01753, 1965.
Frey, H. U., Mende, S. B., and Angelopoulos, A.: Substorm onset observations by IMAGE-FUV, J. Geophys. Res., 109, A10304, doi:10.1029/2004JA010607, 2004.

Grocott, A., Cowley, S. W. H., Sigwarth, J. B., Watermann, J. F., and Yeoman, T. K.: Excitation of twin-vortex flow in the nightside high-latitude ionosphere during an isolated substorm, Ann. Geophys., 20, 1577-1601, doi:10.5194/angeo-20-1577-2002, 2002.

Haaland, S., Lybekk, B., Svenes, K., Pedersen, A., Förster, M., Vaith, H., and Torbert, R.: Plasma transport in the magnetotail lobes, Ann. Geophys., 27, 3577-3590, doi:10.5194/angeo-273577-2009, 2009.

Huang, C.-S., DeJong, A., and Cai, X.: Magnetic flux in the magnetotail and polar cap during sawteeth, isolated substorms, and steady magnetospheric convection events, J. Geophys. Res., 114, A07202, doi:10.1029/2009JA014232, 2009.

Hubert, B., Milan, S. E., Grocott, A., Blockx, C., Cowley, S. W. H., and Gérard, J.-C.: Dayside and nightside reconnection rates inferred from IMAGE FUV and Super Dual Auroral Radar Network data, J. Geophys. Res., 111, A03217, doi:10.1029/2005JA011140, 2006a.

Hubert, B., Palmroth, M., Laitinen, T. V., Janhunen, P., Milan, S. E., Grocott, A., Cowley, S. W. H., Pulkkinen, T., and Gérard, J.-C.: Compression of the Earth's magnetotail by interplanetary shocks directly drives transient magnetic flux closure, Geophys. Res. Lett., 33, L10105, doi:10.1029/2006GL026008, 2006b.

Hubert, B., Kauristie, K., Amm, O., Milan, S. E., Grocott, A., Cowley, S. W. H., and Pulkkinen, T. I.: Auroral streamers and magnetic flux closure, Geophys. Res. Lett., 34, L15105, doi:10.1029/2007GL030580, 2007.

Hubert, B., Milan, S. E., Grocott, A., Cowley, S. W. H., and Gérard, J.-C.: Open magnetic flux and magnetic flux closure during sawtooth events, Geophys. Res. Lett., 35, L23301, doi:10.1029/2008GL036374, 2008.

Hubert, B., Blockx, C., Milan, S. E., and Cowley, S. W. H.: Statistical properties of flux closure induced by solar wind dynamic pressure fronts, J. Geophys. Res., 114, A07211, doi:10.1029/2008JA013813, 2009.

Hubert, B., Aikio, A. T., Amm, O., Pitkänen, T., Kauristie, K., Milan, S. E., Cowley, S. W. H., and Gérard, J.-C.: Comparison of the open-closed field line boundary location inferred using IMAGEFUV SI12 images and EISCAT radar observations, Ann. Geophys., 28, 883-892, doi:10.5194/angeo-28-883-2010, 2010.

Juusola, L., Partamies, N., and Tanskanen, E.: Effect of the ring current on preconditioning the magnetosphere for steady magnetospheric convection, Geophys. Res. Lett., 40, 1917-1921, doi:10.1002/grl.50405, 2013.

Kissinger, J., McPherron, R. L., Hsu, T.-S., Angelopoulos, V., and Chu, X.: Necessity of substorm expansions in the initiation of steady magnetospheric convection, Geophys. Res. Lett., 39, L15105, doi:10.1029/2012GL052599, 2012a.

Kissinger, J., McPherron, R. L., Hsu, T.-S., and Angelopoulos, V.: Diversion of plasma due to high pressure in the inner magnetosphere during steady magnetospheric convection, J. Geophys. Res., 117, A05206, doi:10.1029/2012JA017579, 2012 b.

Kivelson, M. G. and Russel, C. T.: Introduction to Space Physics, Cambridge University Press, 1997.

Mann, I. R., Milling, D. K., Rae, I. J., Ozeke, L. G., Kale, A., Kale, Z. C., Murphy, K. R., Parent, A., Usanova, M., Pahud, D. M., Lee, E.-A., Amalraj, V., Wallis, D. D., Angelopoulos, V., Glass- 
meier, K.-H., Russell, C. T., Auster, H.-U., and Singer, H. J.: The upgraded CARISMA magnetometer array in the THEMIS era, Space Sci. Rev., 141, 413-451, doi:10.1007/s11214-008-9457-6, 2008 (data available at: http://www.carisma.ca/).

McPherron, R. L.: Growth phase of magnetospheric substorms, J. Geophys. Res. 75, 5592-5599, 1970.

McWilliams, K. A., Pfeifer, J. B., McPherron, R. L., and Frey, H. U.: SuperDARN and IMAGE WIC observations during intervals of steady magnetospheric convection, Proc. Int. Conf. Substorms-8, 27-31 March 2006, 175-180, 2006.

Mende, S. B., Heetderks, H., Frey, H. U., Lampton, M., Geller, S. P., Habraken, S., Renotte, E., Jamar, C., Rochus, P., Spann, J., Fuselier, S. A., Gérard, J. C., Gladstone, G. R., Murphree, S., and Cogger, L.: Far ultraviolet imaging from the IMAGE spacecraft: 1. System design, Space Sci. Rev., 91, 243-270, 2000a.

Mende, S. B., Heetderks, H., Frey, H. U., Stock, J. M., Lampton, M., Geller, S., Abiad, R., Siegmund, O., Habraken, S., Renotte, E., Jamar, C., Rochus, P., Gérard, J. C., Sigler, R., and Lauche, H.: Far ultraviolet imaging from the IMAGE spacecraft: 3. Spectral imaging of Lyman alpha and OI $135.6 \mathrm{~nm}$, Space Sci. Rev., 91, 287-318, 2000b.

Milan, S. E.: A simple model of the flux content of the distant magnetotail, J. Geophys. Res., 109, A07210, doi:10.1029/2004JA010397, 2004.

Milan, S. E., Lester, M., Cowley, S. W. H., Oksavik, K., Brittnacher, M., Greenwald, R. A., Sofko, G., and Villain, J.-P.: Variations in the polar cap area during two substorm cycles, Ann. Geophys., 21, 1121-1140, doi:10.5194/angeo-21-1121-2003, 2003.

Milan, S. E., Cowley, S. W. H., Lester, M., Wright, D. M., Slavin, J. A., Fillingim, M., Carlson, C. W., and Singer, H. J.: Response of the magnetotail to changes in the open flux content of the magnetosphere, J. Geophys. Res., 109, A04220, doi:10.1029/2003JA010350, 2004.

Milan, S. E., Provan, G., and Hubert, B.: Magnetic flux transport in the Dungey cycle: A survey of dayside and nightside reconnection rates, J. Geophys. Res., 112, A01209, doi:10.1029/2006JA011642, 2007.

Milan, S. E., Grocott, A., Forsyth, C., Imber, S. M., Boakes, P. D., and Hubert, B.: A superposed epoch analysis of auroral evolution during substorm growth, onset and recovery: open magnetic flux control of substorm intensity, Ann. Geophys., 27, 659-668, doi:10.5194/angeo-27-659-2009, 2009.

O'Brien, T. P., Thomson, S. M., and Mc Pherron, R. L.: Steady magnetospheric convection: Statistical signatures in the solar wind and AE, Geophys. Res. Lett., 29, 1130, doi:10.1029/2001GL014641, 2002.

Partamies, N., Pulkkinen, T. I., McPherron, R. L., McWilliams, K., Bryant, C. R., Tanskanen, E., Singer, H. J., Reeves, G. D., and Thomsen, M. F.: Statistical survey on sawtooth events, SMCs and isolated substorms, Adv. Space Res., 44, 376-384, doi:10.1016/j.asr.2009.03.013, 2009a.
Partamies, N., Pulkkinen, T. I., McPherron, R. L., McWilliams, K., Bryant, C. R., Tanskanen, E., Singer, H. J., Reeves, G. D., and Thomsen, M. F.: Different magnetospheric modes: solar wind driving and coupling efficiency, Ann. Geophys., 27, 4281-4291, doi:10.5194/angeo-27-4281-2009, 2009b.

Petrinec, S. M. and Russell, C. T.: An empirical model of the size and shape of the near-Earth magnetotail, Geophys. Res. Lett., 20, 2695-2698, 1993.

Petrinec, S. M. and Russell, C. T.: Near-Earth magnetotail shape and size as determined from the magnetopause flaring angle, J. Geophys. Res., 101, 137-152, 1996.

Ruohoniemi, J. M., Greenwald, R. A., Baker, K. B., Villain, J. P., and McCready, M. A.: Drift motions of small-scale irregularities in the high-latitude F region: An experimental comparison with plasma drift motions, J. Geophys. Res., 92, 45534564, doi:10.1029/JA092iA05p04553, 1987 (data available at: http://superdarn.jhuapl.edu/).

Sergeev, V. A. and Lennartsson, W.: Plasma sheet at X -20 RE during steady magnetospheric convection, Planet. Space Sci., 36, 353-370, 1988.

Sergeev, V. A., Pellinen, R. J., and Pulkkinen, T. I.: Steady magnetic convection: a review of recent results, Space. Sci. Rev., 75, 551604, 1996.

Siscoe, G. L. and Huang, T. S.: Polar cap inflation and deflation, J. Geophys. Res., 90, 543-547, 1985.

Stone, E. C., Frandsen, A. M., Mewaldt, R. A., Christian, E. R., Margolies, D., Ormes, J. F., and Snow, F.: The Advanced Composition Explorer, Space Sci. Rev., 86, 1-22, doi:10.1023/A:1005082526237, 1998 (data available at: https: //cdaweb.gsfc.nasa.gov/sp_phys/).

Tanskanen, E. I., Slavin, J. A., Fairfield, D. H., Sibeck, D. G., Gjerloev, J., Mukai, T., Ieda, A., and Nagai, T.: Magnetotail response to prolonged southward IMF Bz intervals: Loading, unloading, and continuous magnetospheric dissipation, J. Geophys. Res., 110, A03216, doi:10.1029/2004JA010561, 2005.

Viljanen, A. and Häkkinen, L.: IMAGE magnetometer network, in: Satellite-ground Based Coordination Sourcebook, edited by: Lockwood, M., Wild, M. N. and Opgenoorth, H. J., 111-117, Eur. Space Agency Spec. Publ., ESA-SP, 1198, Fennoscandia and Svalbard, 1997 (data available at: http://space.fmi.fi/image/ beta/).

Walach, M.-T. and Milan, S. E.: Are steady magnetospheric convection events prolonged substorms?, J. Geophys. Res.-Space, 120, 1751-1758, doi:10.1002/2014JA020631, 2015.

Yahnin, A., Malkov, M. V., Sergeev, V. A., Pellinen, R. J., Aulamo, O., Vennergstrom, S., Friis-Christensen, E., Lassen, K., Danielsen, C., and Craven, J. D.: Features of steady magnetospheric convection, J. Geophys. Res., 99, 4039-4051, doi:10.1029/93JA02868, 1994. 